\title{
1. Numerical Modelling of consolidation-induced solute transport in 2 unsaturated soil with dynamic hydraulic conductivity and degree of saturation
}

\author{
$\mathrm{S} \mathrm{Wu}^{1,2}$, D-S Jeng ${ }^{1,2, \#}$, B R Seymour ${ }^{3}$ \\ ${ }^{1}$ School of Civil Engineering, Qingdao University of Technology, Qingdao, 266033, China \\ ${ }^{2}$ School of Engineering \& Built Environment, Griffith University Gold Coast Campus, QLD 4222, Australia \\ ${ }^{3}$ Department of Mathematics, The University of British Columbia, Vancouver, V6T 1Z2, Canada \\ \# Corresponding author.d.jeng@Griffith.edu.au (DSJ)
}

\section{Abstract}

The effects of the pore pressure related dynamic hydraulic conductivity and dynamic degree of saturation on the consolidation-induced solute transport in deformable unsaturated soils are investigated. The storage equation and solute transport equations are revised to account for the dependence of these two soil parameters on pore pressures. Three dynamic models were conducted for dynamic hydraulic conductivity, dynamic degree of saturation, and both. Compared with the conventional model, the simulation results showed that both hydraulic conductivity and degree of saturation increased near the soil surface where pore pressure exceeded air-entry value. Dynamic hydraulic conductivity results in a slightly slower solute transport while dynamic degree of saturation accelerates the migration of contaminants. Including both dynamic effects produced limited differences in solute concentration, while consolidation results were affected significantly. Although dynamic degree of saturation has some influences on consolidation-induced solute transport, the correlation of the dynamic path is less significant. The air-entry value is an important parameter to determine when soil parameters become dynamic, and it affects the soil consolidation process in certain extent. This study provides new methods to incorporate in-homogeneous soil parameters, which are not only spatiotemporal variables, but also dynamic variations with pore pressure.

Keywords: Dynamic hydraulic conductivity; dynamic degree of saturation; solute transport; consolidation; unsaturated soil; deformable soil. 


\section{Introduction}

Contaminant mass transport through porous media is usually described by well-established conventional transport models $[1,2]$ with the ability to account for advection, dispersion, and sorption. Since the mid-20th century, researchers have studied the advection-dispersion equation (ADE) through analytical approximations [3], numerical simulations $[4,5]$, and experimental investigations [6]. These studies were based on the assumption of a rigid porous media where the volume of the porous media does not change. As a result, the advective flow was usually induced by external hydraulic gradient (ground water flow, rainfall infiltration, etc.), however, considerations of transient flow due to soil deformation was missing. In fact, soil volume change (i.e., soil consolidation) occurs simultaneously with solute transport in many cases. For example, it occurs where the field is under an applied load (self-weight, fill placement, finite size loading, etc.) or experiencing changes in the groundwater table (pumping, artesian wells, etc.). In such cases, the coupled effect of soil deformation and solute transport would be necessary to be included in modelling. Alshawabkeh et al.[7] showed that the excess pore pressure dissipation produced a transient advective flux of contaminants, that had a strong influence on overall flux. The first attempt at explaining consolidation-induced solute transport in a porous medium was formulated by Potter et al.[8]. Smith [9] derived a one-dimensional(1D) analytical solution for a steady-state concentration with a varying porosity in a fully saturated deformable porous medium. In this model, soil consolidation was incorporated into the solute transport model with the following steps: (1) computing the porosity variation during consolidation, and (2) introducing solute migration into the solid phase. To link two components of solute in both pore fluid and solid phases, a linear sorption relationship was postulated. Peter \& Smith[10] extended the previous model [9] to accommodate the time-dependent solute migration process. Zhang et al.[12] using the consolidation theory [11], further developed the small strain model [10] to account for a degree of saturation and fluid compressibility. Pu \& Fox[13] used a piecewise method to study containment transport in a saturated layered soil. To incorporate the partially saturated soil environment, Wu \& Jeng [14] conducted a comprehensive parametric study to examine the effect of soil stratification on consolidation-induced solute transportation and demonstrated the importance of correctly modelling a multi-layered soil instead of simply assuming the averaged properties across the soil profile. 
Previous studies have treated the hydraulic conductivity and the degree of saturation as constant in the entire soil layer. However, hydraulic conductivity has been reported to be a function of void ratio $(e)[15,16,17]$. Further, pore-water pressure also affects the hydraulic conductivity $[18,19,20]$. Changes in pore water pressure lead to changes in stresses and in turn soil deformation. Since the hydraulic properties of the soil, such as porosity, hydraulic conductivity and water storage capacity are affected by the changes in stresses, so too will the void flow. This coupled effect highlights the strong correlation between flow and stress-deformation, and the connection to the effects of pore pressure related hydraulic conductivity and the degree of saturation to the consolidation-induced solute transportation. Different expressions for the constitutive relationships of dynamic hydraulic conductivity and degree of saturation were available such as presented in $[20,21,23]$.

The aim of this study is to investigate the effects of dynamic hydraulic conductivity and the degree of saturation on consolidation-induced solute transport in unsaturated deformable porous media. First, these effects are introduced when there is positive pore pressure, then new nonlinear governing equations for the pore pressure field and solute concentration field are derived. Finally, parametric studies for solute transport in an unsaturated soil layer are conducted to investigate the influence of dynamic parameters on the consolidation-induced solute transport.

\section{Dynamic hydraulic conductivity and degree of saturation as functions of pore pressures}

Dynamic hydraulic conductivity and degree of saturation are considered to be functions of pore pressures in order to investigate the consolidation-induced solute transport in a deformable medium. Herein, some key previous theories are outlined and the modified forms of this dependence are proposed.

\subsection{Dynamic hydraulic conductivity}

Several different forms of the dependence of hydraulic conductivity $(K(p))$ on the pore pressure $p$ (or equivalently suction $s$ ) have been proposed. Gardner [20] considered two forms for hydraulic conductivity, a power law of the form:

$$
K(s)=\frac{a}{s^{d}+b},
$$


for various $d$, and an exponential law:

$$
K(s)=a e^{-f s} \text {, or } K(p)=a e^{f p} .
$$

\subsection{Dynamic degree of saturation}

For the degree of saturation, Gallipoli[23], using experimental data, proposed a model of the form:

$$
S_{r}(s)=\left(\frac{1}{1+(\gamma s)^{m_{1}}}\right)^{m_{2}},
$$


in which $\gamma, m_{1}, m_{2}$ are soil constants.

Wu \& Zhang [21] proposed an exponential relationship between degree of saturation and pore pressure in the same format as they used for hydraulic conductivity. The dynamic degree of saturation was determined with respect to suction:

$$
S_{r}(s)= \begin{cases}1 & |s| \leq\left|\psi_{a e}\right|, \\ e^{\alpha \psi_{a e}+\alpha p} & |s|>\left|\psi_{a e}\right|,\end{cases}
$$

With loads acting on the soil matrix, air bubbles may be expelled out from the pore fluid, leading to an increase in the degree of saturation. However, $S_{r}(p)$ cannot exceed unity. To incorporate the positive pore pressure, the constitutive relationship for dynamic degree of saturation is proposed as:

$$
S_{r}(p)= \begin{cases}S_{r}^{0} & |p| \leq\left|\psi_{a e}\right|, \\ -e^{\alpha \psi_{a e}-\alpha p}+1+S_{r}^{0} & \left|\psi_{a e}\right|<|p| \leq\left|P_{S r=1}\right|, \\ 1 & |p|>\left|P_{S r=1}\right| .\end{cases}
$$

The degree of saturation $\left(S_{r}(p)\right)$ is again picked to be related to the air-entry value $\psi_{a e}[\mathrm{kPa}]$ and the de-saturation value $\alpha[1 / \mathrm{kPa}] ; S_{r}^{0}$ refers to the initial degree of saturation (or commonly known as the residual saturation) before a load acts on the soil. Fig. 1 illustrates the relationship between dynamic degree of saturation and the pore pressure (6), but not to scale. In the figure, another important parameter $P_{S r=1}$ is defined as that pore pressure at which $S_{r}$ reaches unity. Beyond this point, $S_{r}$ will stop increasing; i.e., the soil matrix remains fully saturated.

When $S_{r}$ is dynamic, it will vary both spatially and temporally. The variation of degree of saturation is particular important in deriving the consolidation-induced solute transport theory. Herein, the partial derivative of $S_{r}$ with respect to $p$ will be used and is firstly introduced here:

$$
\frac{d S_{r}(p)}{d p}= \begin{cases}0 & |p| \leq\left|\psi_{a e}\right| \\ \alpha e^{\alpha \psi_{a e}-\alpha p} & \left|\psi_{a e}\right|<|p| \leq\left|P_{S r=1}\right| \\ 0 & |p|>\left|P_{S r=1}\right|\end{cases}
$$




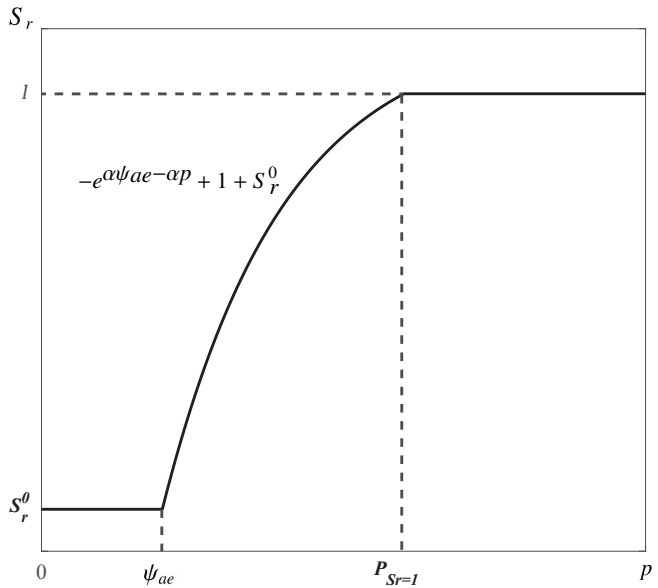

(a) Dynamic degree of saturation

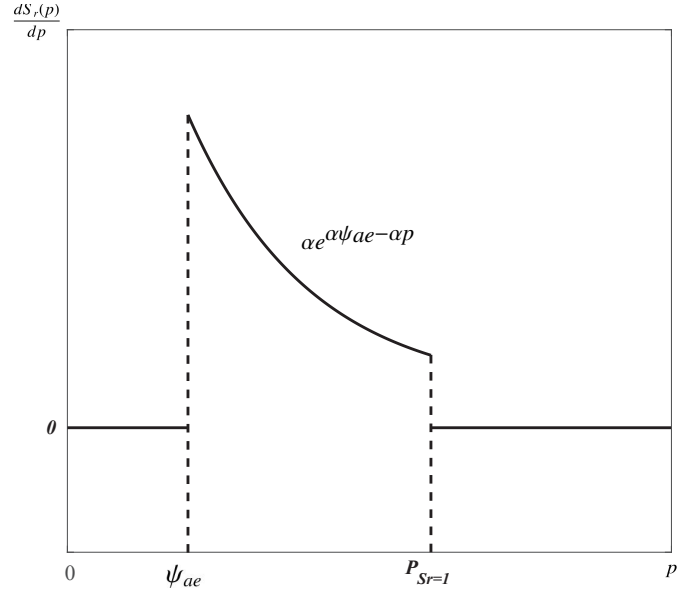

(b) Partial differentiation of degree of saturation

Figure 1: Relationship between the dynamic degree of saturation and pore pressure (Type 1)

and the co-responding derivative of $S_{r}$ with respect to $p$ becomes:

$$
\frac{d S_{r}(p)}{d p}= \begin{cases}0 & |p| \leq\left|\psi_{a e}\right|, \\ \alpha e^{\alpha \psi_{a e}+\alpha p} & \left|\psi_{a e}\right|<|p| \leq\left|P_{S r=1}\right| \\ 0 & |p|>\left|P_{S r=1}\right| .\end{cases}
$$




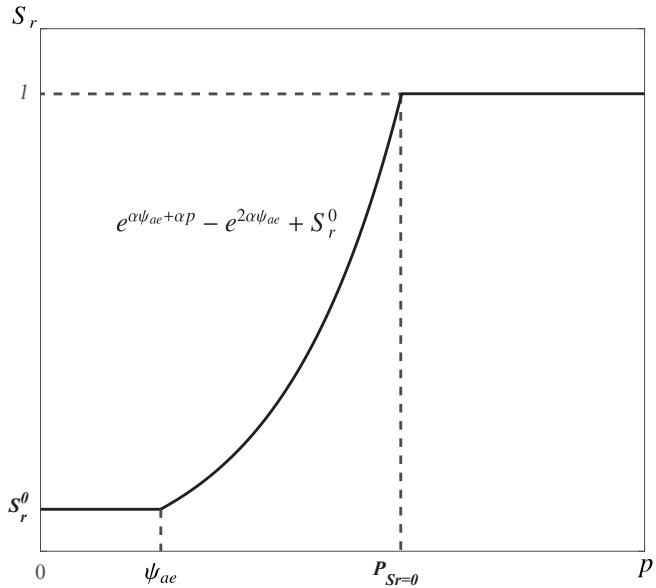

(a) Dynamic degree of saturation

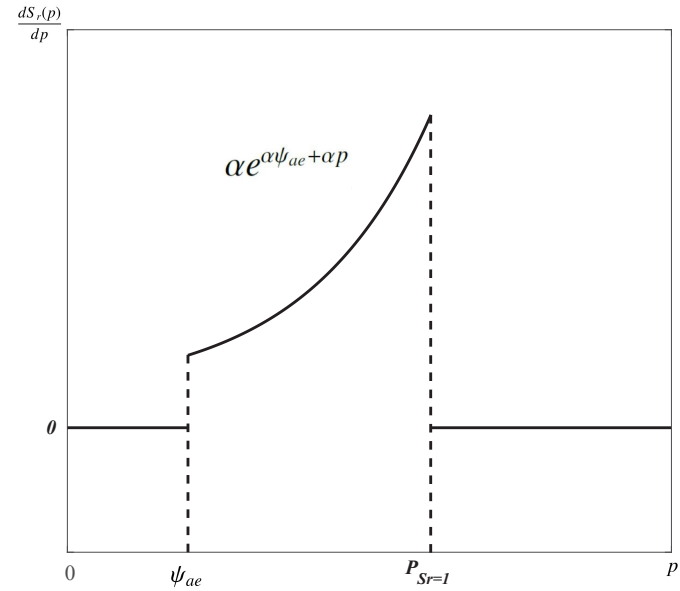

(b) Partial differentiation of degree of saturation

Figure 2: Relationship between the dynamic degree of saturation and pore pressure (Type 2)

The diagram for the dynamic degree of saturation relationship with pore pressure for Type 2 is shown in Fig. 2. As for Type 1, the degree of saturation increases from 0.85 to unity as the pore pressure increases. For Type 2 the $S_{r}$ versus $p$ curve is convex, which results in a increasing function $\frac{d S_{r}(p)}{d p}$.

\section{Theoretical models}

When an external load is applied to a deformable soil matrix, an excess pore pressure is generated. Under draining conditions, the excess pore pressures will gradually dissipate after the load stops increasing (post-loading stage). When there is a source of non-active pollution, the contaminants are carried by the transient advective flow (triggered by the excess pore pressure) and finally spread and cause pollution. To model this process, three governing equations are required to calculate the excess pore pressure, soil displacement and solute concentration fields.

In this study, four models are considered, with summary shown in Table 2. Note that, for both Model Srp and Model Kp+Srp, dynamic degree of saturation formulation Type 1 is used. The following sections explain the theories of the conventional model and modified dynamic models.

Here the work of Zhang et al.[12] is extended to consider the effects of dynamic hydraulic conductivity and degree of saturation. Although the original equilibrium equation is directly applicable to all dynamic models, the governing equations for both the excess pore pressures 


\begin{tabular}{|c|c|c|}
\hline Name in paper & Label in figures & Description \\
\hline Conventional model & $\mathrm{CON}$ & $\begin{array}{l}\text { coupled consolidation-transport model, with } \\
\text { constant } K \text { and } S_{r} \text {, similar as [12]. }\end{array}$ \\
\hline Dynamic $K$ model & $\mathrm{Kp}$ & $\begin{array}{l}\text { coupled consolidation-transport model, with } \\
\text { constitutive law for } K(p), \mathrm{Eq}(3) \text {, and constant } \\
S_{r}\end{array}$ \\
\hline Dynamic $S_{r}$ model & Srp & $\begin{array}{l}\text { coupled consolidation-transport model, with } \\
\left.\text { constitutive law for } S_{r}(p) \text { (Type } 1, \mathrm{Eq}(6)\right) \text { and } \\
\text { constant } K\end{array}$ \\
\hline Dynamic $K \& S_{r}$ model & $\mathrm{Kp}+\mathrm{Srp}$ & $\begin{array}{l}\text { coupled consolidation-transport model, with } \\
\text { constitutive laws for } K(p), \mathrm{Eq} \text { (3) and } \\
\left.S_{r}(p) \text { (Type } 1, \mathrm{Eq}(6)\right)\end{array}$ \\
\hline
\end{tabular}

and concentration fields need to be updated. This section will only present the final governing equations for each model while the detailed derivation is in Appendixes A \& B.

According to [12], the force balance equation is:

$$
G \frac{2(1-v)}{(1-2 v)} \frac{\partial^{2} u}{\partial z^{2}}+\left(1-n^{0}\right)\left(\rho_{s}-S_{r} \rho_{w}\right) g \frac{\partial u}{\partial z}=\frac{\partial p}{\partial z}
$$

where $u[\mathrm{~m}]$ is the vertical displacement, $p[\mathrm{~Pa}]$ is the excess pore pressure, $G[\mathrm{~Pa}]$ is the shear modulus and assumed to be constant in this study, $v$ is the Poisson's ratio, and $\rho_{w}\left[\mathrm{~kg} / \mathrm{m}^{3}\right] \& \rho_{s}$ $\left[\mathrm{kg} / \mathrm{m}^{3}\right]$ denote the densities of the pore fluid and solid particles.

\subsection{Dynamic K\&Sr model}

The spatial derivative of hydraulic conductivity for $|p|>\left|\psi_{a e}\right|$ is: 


$$
\frac{\partial K}{\partial z}=\frac{d K}{d p} \frac{\partial p}{\partial z}=\alpha K_{s} e^{\alpha \psi_{a e}+\alpha p} \frac{\partial p}{\partial z}=\alpha K \frac{\partial p}{\partial z}
$$

120 121

Similarly, the temporal and spatial derivations of degree of saturation (Type 1) are, for $\left|\psi_{a e}\right|<$ $|p| \leq\left|P_{S r=1}\right|:$

$$
\frac{\partial S_{r}}{\partial t}=\frac{d S_{r}}{d p} \frac{\partial p}{\partial t}=\alpha e^{\alpha \psi_{a e}-\alpha p} \frac{\partial p}{\partial t}
$$

and

$$
\frac{\partial S_{r}}{\partial z}=\frac{d S_{r}}{d p} \frac{\partial p}{\partial z}=\alpha e^{\alpha \psi_{a e}-\alpha p} \frac{\partial p}{\partial z}
$$

It is important to point out that, by using product law, the term $\frac{\partial K}{\partial t}, \frac{\partial S_{r}}{\partial t}, \frac{\partial S_{r}}{\partial z}$ could be solved as long as $\frac{d K}{d p}$ and $\frac{d S_{r}}{d p}$ are known. Therefore, other forms of dynamic constitutive relationships are also applicable.

Substitute (11)-(13) into (A.11), and replace porosity $n$ with its initial value $n^{0}$ [12], then the governing equations for excess pore pressure are summarised as:

- for $|p| \leq\left|\psi_{a e}\right|$

$$
S_{r} n^{0} \beta \frac{\partial p}{\partial t}+S_{r} \frac{\partial^{2} u}{\partial z \partial t}=\frac{K}{\rho_{w} g}\left(\frac{\partial^{2} p}{\partial z^{2}}\right)
$$

where $\beta$ is the fluid compressibility as defined in (A.5) [27].

- for $\left|\psi_{a e}\right|<|p| \leq\left|P_{S r=1}\right|$,

$$
\left(S_{r} n^{0} \beta+n^{0} \alpha e^{\alpha \psi_{a e}-\alpha p}\right) \frac{\partial p}{\partial t}+S_{r} \frac{\partial^{2} u}{\partial z \partial t}=\frac{K}{\rho_{w} g}\left(\frac{\partial^{2} p}{\partial z^{2}}\right)+\frac{\alpha K}{\rho_{w} g}\left(\frac{\partial p}{\partial z}\right)^{2}-n^{0} \alpha e^{\alpha \psi_{a e}-\alpha p} \frac{\partial u}{\partial t} \frac{\partial p}{\partial z}
$$

- for $|p|>\left|P_{S r=1}\right|$,

$$
S_{r} n^{0} \beta \frac{\partial p}{\partial t}+S_{r} \frac{\partial^{2} u}{\partial z \partial t}=\frac{K}{\rho_{w} g}\left(\frac{\partial^{2} p}{\partial z^{2}}\right)+\frac{\alpha K}{\rho_{w} g}\left(\frac{\partial p}{\partial z}\right)^{2} .
$$

The governing equations for solute transport with dynamic hydraulic conductivity and degree of saturation are given below, with the detailed derivations provided in Appendix B. 
- for $|p| \leq\left|\psi_{a e}\right|$ or $|p|>\left|P_{S r=1}\right|$,

$$
\begin{aligned}
{\left[S_{r} n^{0}+\left(1-n^{0}\right) \rho_{s} K_{d}\right] \frac{\partial c}{\partial t} } & =\frac{\partial^{2} c}{\partial z^{2}}\left(S_{r} n^{0} D_{m}-\alpha_{L} \frac{K}{\rho_{w} g} \frac{\partial p}{\partial z}\right) \\
& +\frac{\partial c}{\partial z}\left\{-\alpha_{L} S_{r} n^{0} \beta \frac{\partial p}{\partial t}-\alpha_{L} S_{r} \frac{\partial^{2} u}{\partial z \partial t}\right. \\
& +\frac{\alpha_{L} \beta K}{\rho_{w} g}\left(\frac{\partial p}{\partial z}\right)^{2}+S_{r} D_{m}\left(1-n^{0}\right) \frac{\partial^{2} u}{\partial z^{2}} \\
& \left.+\frac{K}{\rho_{w} g} \frac{\partial p}{\partial z}-\left[S_{r} n^{0}+\left(1-n^{0}\right) \rho_{s} K_{d}\right] \frac{\partial u}{\partial t}\right\} \\
& +c\left[S_{r} n^{0} \beta \frac{\partial p}{\partial t}-\beta \frac{K}{\rho_{w} g}\left(\frac{\partial p}{\partial z}\right)^{2}+S_{r} n^{0} \beta \frac{\partial u}{\partial t} \frac{\partial p}{\partial z}\right],
\end{aligned}
$$

- for $\left|\psi_{a e}\right|<|p| \leq\left|P_{S r=1}\right|$

$$
\begin{aligned}
{\left[S_{r} n^{0}+\left(1-n^{0}\right) \rho_{s} K_{d}\right] \frac{\partial c}{\partial t} } & =\frac{\partial^{2} c}{\partial z^{2}}\left(S_{r} n^{0} D_{m}-\alpha_{L} \frac{K}{\rho_{w} g} \frac{\partial p}{\partial z}\right) \\
& +\frac{\partial c}{\partial z}\left\{n^{0} \alpha e^{\alpha \psi_{a e}-\alpha p}\left(D_{m} \frac{\partial p}{\partial z}-\alpha_{L} \frac{\partial p}{\partial t}-\alpha_{L} \frac{\partial u}{\partial t} \frac{\partial p}{\partial z}\right)\right. \\
& -\alpha_{L} S_{r} n^{0} \beta \frac{\partial p}{\partial t}-\alpha_{L} S_{r} \frac{\partial^{2} u}{\partial z \partial t} \\
& +\frac{\alpha_{L} \beta K}{\rho_{w} g}\left(\frac{\partial p}{\partial z}\right)^{2}+S_{r} D_{m}\left(1-n^{0}\right) \frac{\partial^{2} u}{\partial z^{2}} \\
& \left.+\frac{K}{\rho_{w} g} \frac{\partial p}{\partial z}-\left[S_{r} n^{0}+\left(1-n^{0}\right) \rho_{s} K_{d}\right] \frac{\partial u}{\partial t}\right\} \\
& +c\left[S_{r} n^{0} \beta \frac{\partial p}{\partial t}-\beta \frac{K}{\rho_{w} g}\left(\frac{\partial p}{\partial z}\right)^{2}+S_{r} n^{0} \beta \frac{\partial u}{\partial t} \frac{\partial p}{\partial z}\right]
\end{aligned}
$$

\subsection{Dynamic Sr model}

When the degree of saturation is dynamic (Type 1) and the hydraulic conductivity is constant, the governing equations for excess pore pressure field are:

- for $|p| \leq\left|\psi_{a e}\right|$ or $|p|>\left|P_{S r=1}\right|$, same as (14),

- for $\left|\psi_{a e}\right|<|p| \leq\left|P_{S r=1}\right|$

$$
\left(S_{r} n^{0} \beta+n^{0} \alpha e^{\alpha \psi_{a e}-\alpha p}\right) \frac{\partial p}{\partial t}+S_{r} \frac{\partial^{2} u}{\partial z \partial t}=\frac{K}{\rho_{w} g}\left(\frac{\partial^{2} p}{\partial z^{2}}\right)-n^{0} \alpha e^{\alpha \psi_{a e}-\alpha p} \frac{\partial u}{\partial t} \frac{\partial p}{\partial z} .
$$

The updated solute transport equations are:

- for $|p| \leq\left|\psi_{a e}\right|$ or $|p|>\left|P_{S r=1}\right|$, same as (17)

- for $\left|\psi_{a e}\right|<|p| \leq\left|P_{S r=1}\right|$, same as (18) 


\subsection{Dynamic K model}

Under the assumption of dynamic hydraulic conductivity but constant degree of saturation, the governing equations for excess pore pressure are:

- for $|p| \leq\left|\psi_{a e}\right|$, same as (14),

- for $|p|>\left|\psi_{a e}\right|$, same as (16).

Although the hydraulic conductivity is dynamic, the solute transport equation is the same as (17). This is because the dynamic hydraulic conductivity term is only introduced into the solute transport equation when replacing the velocity terms. A detailed explanation is given in Appendix B.

\subsection{The conventional model with constant $K$ and $S r$}

When both the hydraulic conductivity and degree of saturation are constant, the governing equation for excess pore pressure is the same as (14), and for solute concentration field, it is written as the form of (17). It is important to point out that these two equations are the same as presented in [12].

\subsection{Model validation}

Since no experimental data for solute transport in a deformable porous medium with dynamic hydraulic conductivity and degree of saturation is available in the literature, a possible validation of the present model is to compare with the previous models with constant soil properties $[10,12]$. To compare with those results, the new dynamic model (with both $K \& S_{r}$ dynamic) was run with a very high $\psi_{a e}$ value $(1000 \mathrm{kPa})$. Then, pore pressure will never exceed

the air-entry value. That is, the dynamic model was actually calculating the conventional study case where both $K$ and $S_{r}$ remains unchanged. Furthermore, utilizing the piecewise function, the ramp load can be applied with different smoothing methods at the turning point. Here, a continuous second derivative was adopted and applied for a period of half a year. This smoothing method can be explained as follows: when the landfill site is about to reach capacity, less waste is disposed into this field, and more waste delivered to a new site. Therefore, the loading rate decreases. Fig. 3 is presented as validation of the newly added smoothing methods. Two 


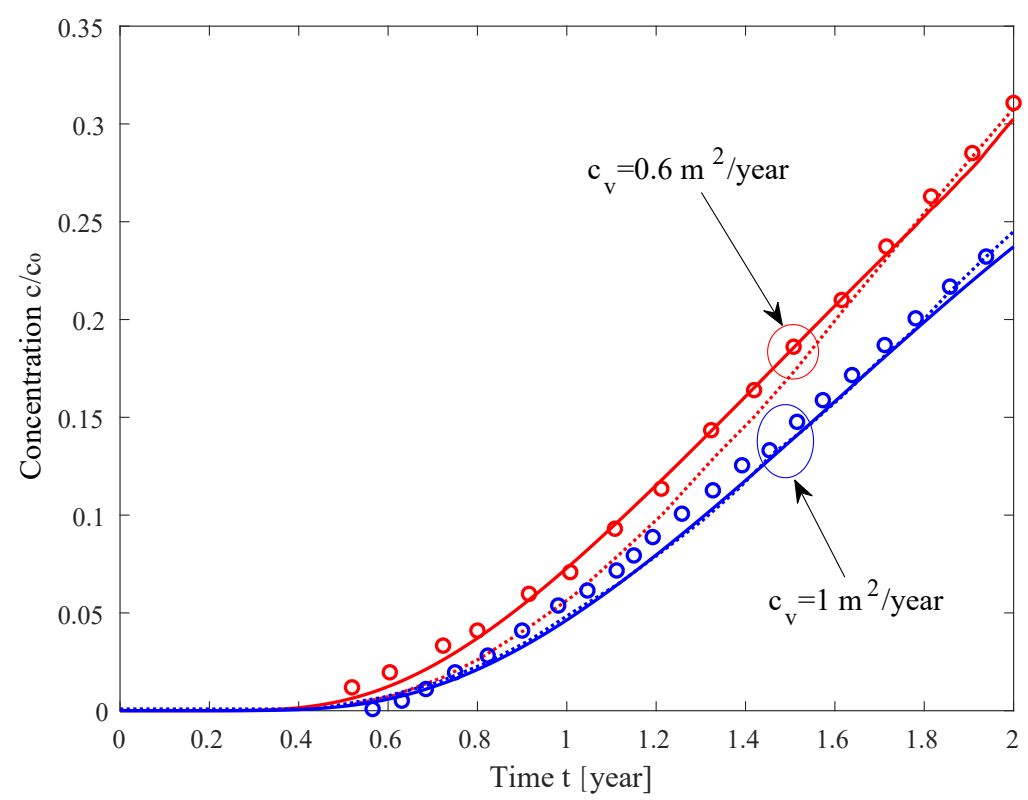

Figure 3: The comparison of the present dynamic model (solid lines) with Zhang et al. (2012) (dotted lines) and Peter and Smith (2002) (circled points). Note: case 1 (red) is presented for $c_{v}=0.6 \mathrm{~m}^{2} / \mathrm{s}$, case 2 (blue) is presented for $c_{v}=1 \mathrm{~m}^{2} / \mathrm{s}$

sets of results were compared with the control of $c_{v}$ calculated from (25). Results in red and blue are for the coefficient of consolidation equals $0.6 \mathrm{~m}^{2} / \mathrm{s}$ and $1.0 \mathrm{~m}^{2} / \mathrm{s}$ respectively. It is clear that the dynamic model with the smoothing method (solid lines) is a good fit to both [12] (dotted lines) and [10] (circled points).

\section{Numerical model for a landfill system}

\subsection{Boundary conditions and initial conditions}

As an application, a landfill with one leachate collection system is assumed to be constructed on the bottom of a compacted clay layer (Fig. 4). The contaminant migration through the clay layer beneath the landfill is evaluated. The width of the landfill site is considered to be larger than the thickness of soil layer, and the load on the top surface is assumed to be uniform, therefore, 1D model will be applied with $z$-axis positive pointing downwards.

At the top boundary $(z=0)$, the impermeable geomembrane layer prevents Darcy's flow. Therefore, a zero excess pore pressure gradient can be postulated, as follows: 


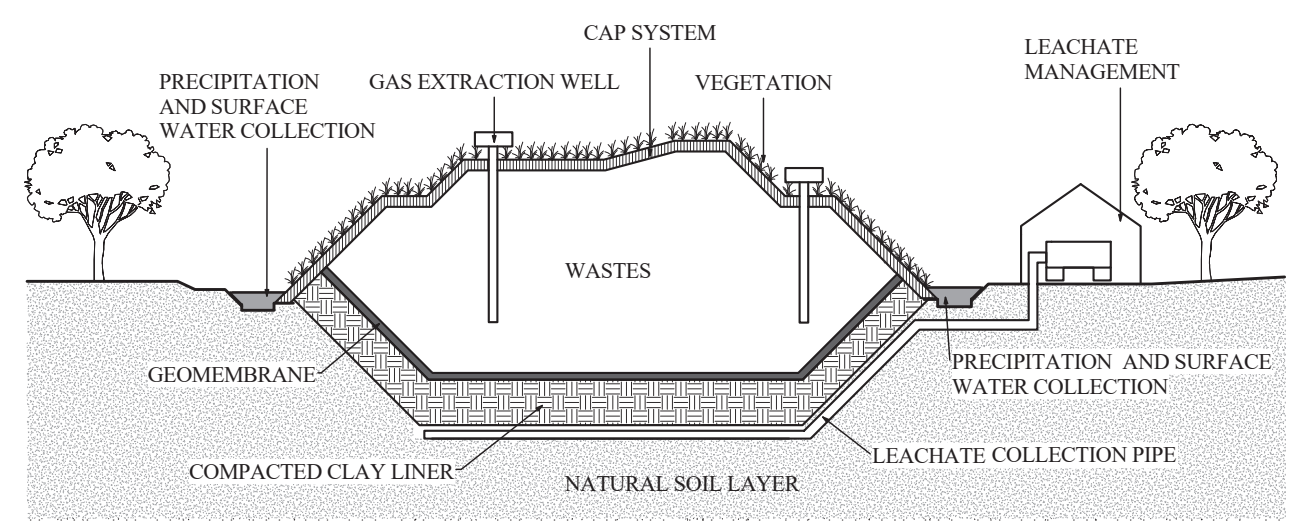

Figure 4: Sketch of a typical landfill site

$$
\frac{\partial p}{\partial z}(0, t)=0
$$

$$
\frac{\partial c}{\partial z}(0, t)=\frac{D_{G}}{n^{0} h D_{m}}\left(c-c_{0}\right),
$$
concentration is expressed as:

According to [12], considering the volatile organic compounds that diffuse through the geomembrane layer and dissolve into the pore water, the top boundary condition for the solute 


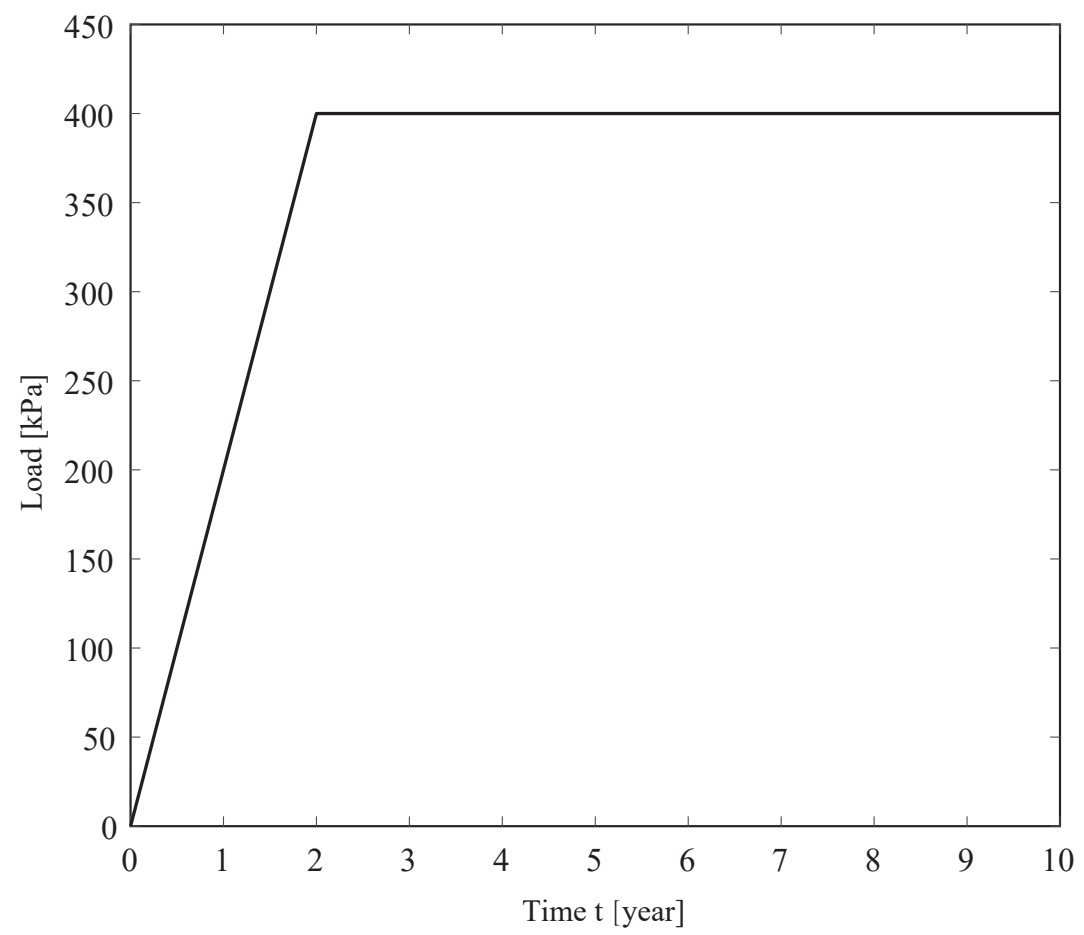

Figure 5: Ramp load

$$
\left\{\begin{array}{c}
p(L, t)=0, \\
u(L, t)=0, \\
\frac{\partial c}{\partial z}(L, t)=0 .
\end{array}\right.
$$

To simplify the model, all initial values for pore pressure field, soil deformation and solute transport are set to zero, i.e.,

$$
\left\{\begin{array}{l}
p(z, 0)=0 \\
u(z, 0)=0 \\
c(z, 0)=0 .
\end{array}\right.
$$


The initial values of dynamic hydraulic conductivity and degree of saturation are also critical in this study, and the initial values $\left(K_{s} \& S_{r}^{0}\right)$ will be introduced in the following section along with other input parameters.

\subsection{Input parameters and numerical approach}

In this study, all models simulates the same landfill case but with different considerations of the hydraulic conductivity and degree of saturation. Table 3 summarises all parameters used in Model CON and parameters only used in the dynamic models.

The combination of the parameters is selected to ensure that the coefficient of consolidation $\left(c_{v}\right)$ stays within the range of $1 \times 10^{-8}$ to $3 \times 10^{-7} \mathrm{~m}^{2} / \mathrm{s}[28,29]$. The coefficient of consolidation $\left(c_{v}\right)$ can be calculated as

$$
c_{v}=\frac{2 G K(1-v)}{\rho_{w} g(1-2 v)} .
$$

Additionally, while making selection of the parameters, soil deformation was kept less than $20 \%$ to satisfy the small deformation assumption.

Note that in the conventional model, $S_{r}$ is assumed to be equal to $S_{r}^{0}$ and $K$ is set to be equal to $K_{s}$ through the whole simulation.

All models are simulated by COMSOL Multiphysics 5.0, which is a popular finite element solver. Some settings such as parametric studies are achieved through COMSOL with Matlab 5.0 .

\section{Results and discussions}

In Section 5.1, 5.2 and 5.3, the results from the dynamic Models $K p, S r p$ and $K p+S r p$ are presented together with the conventional model, CON. Section 5.4 then summarizes the averaged flow velocity and the advective emission for all models. The results in Sections 5.1 - 5.4 are all for the Type $1 S_{r}(p)$ shape. A comparison for the Type $2 S_{r}(p)$ shape is given in Section 5.5 
Table 3: Parameters

\begin{tabular}{lll}
\hline & \multicolumn{2}{c}{ Parameters for Model CON } \\
\hline Parameter & Value & Description \\
\hline$Q(t)$ & As introduced in Section4.1 & Waste loading \\
$h$ & $0.0015 \mathrm{~m}$ & Thickness of geomembrane \\
$L$ & $3 \mathrm{~m}$ & Thickness of clay layer \\
$S_{r}^{0}$ & 0.85 & Initial degree of saturation \\
$n^{0}$ & 0.33 & Initial porosity \\
$G$ & $5 \times 10^{5} \mathrm{~Pa}$ & Shear modulus \\
$v$ & 0.33 & Poisson's ratio \\
$K_{s}$ & $1 \times 10^{-9} \mathrm{~m} / \mathrm{s}$ & Initial hydraulic conductivity \\
$\alpha_{L}$ & $0.1 \mathrm{~m}$ & Longitudianl dispersion factor \\
$\rho_{w}$ & $1 \times 10^{3} \mathrm{~kg} / \mathrm{m}^{3}$ & Initial density of the pore fluid, \\
& & varied due to fluid compressibility \\
$\rho_{s}$ & $2.6 \times 10^{3} \mathrm{~kg} / \mathrm{m}^{3}$ & Density of the solid phase \\
$K_{d}$ & 0 & Partitioning coefficient \\
$r_{h}$ & $0.02 \mathrm{~m}$ & Volumetric fraction of dissolved air \\
& & within pore water \\
$D_{G}$ & $1.5 \times 10^{-4} \mathrm{~m}^{2} / \mathrm{y}$ & Mass transfer coefficient of geomembrane \\
$D_{m}$ & $5 \times 10^{-9} \mathrm{~m}^{2} / \mathrm{s}$ & Molecular diffusion coefficient in the clay \\
$c_{0}$ & $0.1 \mathrm{~kg} / \mathrm{m}^{3}$ & Reference solute concentration \\
$g$ & $9.8 \mathrm{~m} / \mathrm{s}^{2}$ & Gravity acceleration \\
\hline$\psi_{a e}$ & $30 \mathrm{kPa}$ & De-saturation coefficient for clay \\
\hline & $0.016 \mathrm{kPa}{ }^{-1}$ &
\end{tabular}



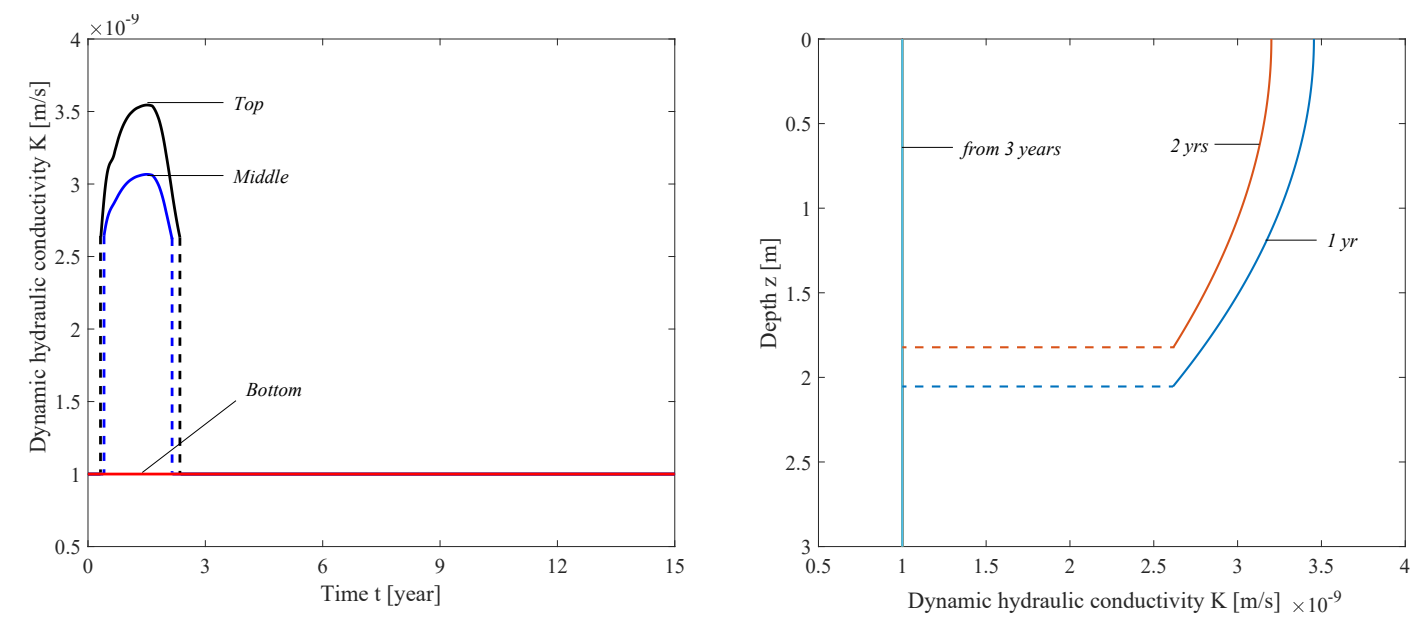

Figure 6: Distribution of dynamic hydraulic conductivity vs time and depth for Model Kp

\subsection{Dynamic hydraulic conductivity model (Model Kp)}

The results presented in this section are from the dynamic hydraulic conductivity model while keeping the degree of saturation as a constant value.

The most important result of Model $\mathrm{Kp}$ is the form of the evolution of the dynamic conductivity $(K)$. Fig. 6 shows changes in $K$ at different locations. At the bottom (the contaminant outlet in the clay layer) $K$ is constant and always equal to $K_{s}$. This is attributed to the zero pore pressure at that point allowing for the pore fluid to flow freely into the leachate collection pipe, since the pore pressure at the outlet will never exceed the air entry value. However, the values of $K$ at both the top point (the contaminant inlet in the clay layer) and the middle point (where $z=L / 2$ in the clay layer) experience increasing and then decreasing periods. The excess pore pressure increases as the ramp load is gradually applied to the clay layer, when the pore fluid does not have time to drain out. Once the pore pressure rises up to the air entry level, $K$ starts to increase. After the load reaches the post-loading period (load stops increasing) in around 2 years, the excess pore pressure starts to dissipate with the pore fluid gradually expelling out from soil matrix. According to the dynamic conductivity model, the pore pressure recovers to the air-entry level, when $K$ then maintains the level of $K_{s}$. The largest $K$ value of around $3.5 \times 10^{-9} \mathrm{~m} / \mathrm{s}$ occurs at the inlet in 1.5 years. The dynamic conductivity curve at the top point is higher than that at the middle point, implying that the pore pressure is generally a maximum at the top. Fig. 6 also shows the distributions of $K$ along the clay layer were plotted at different times. The dark blue line labelled ' $1 y r^{\prime}$ indicates values after 1 year of loading. $K$ curves for 1 

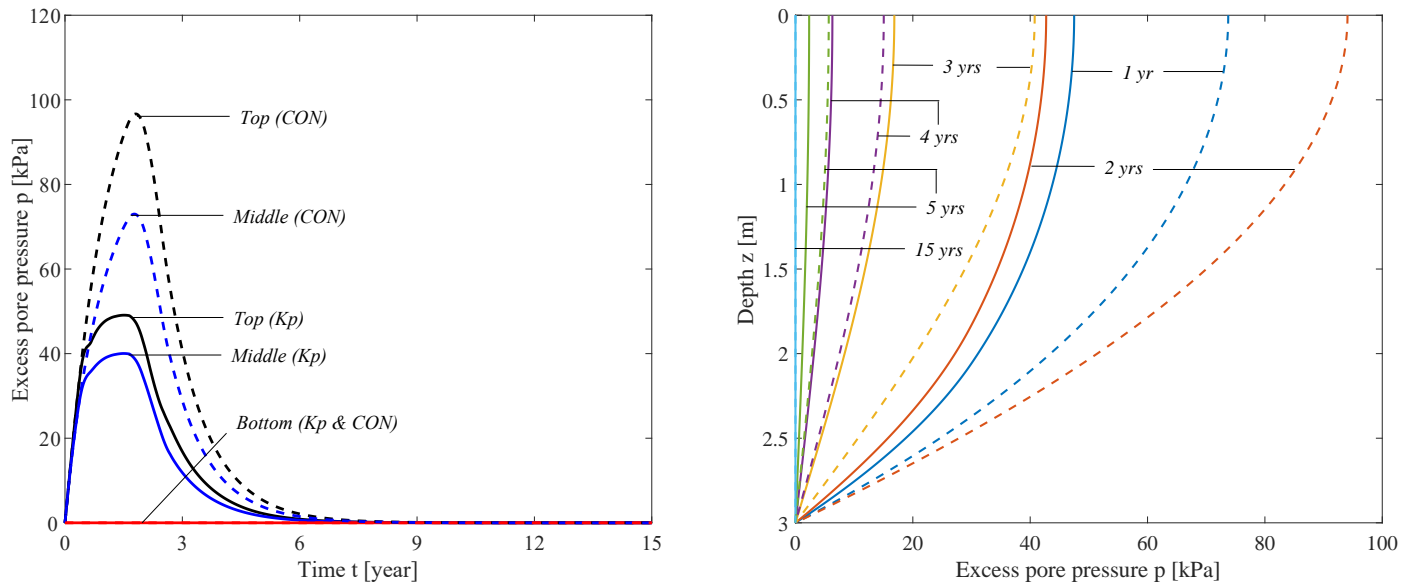

(a) Excess pore pressure
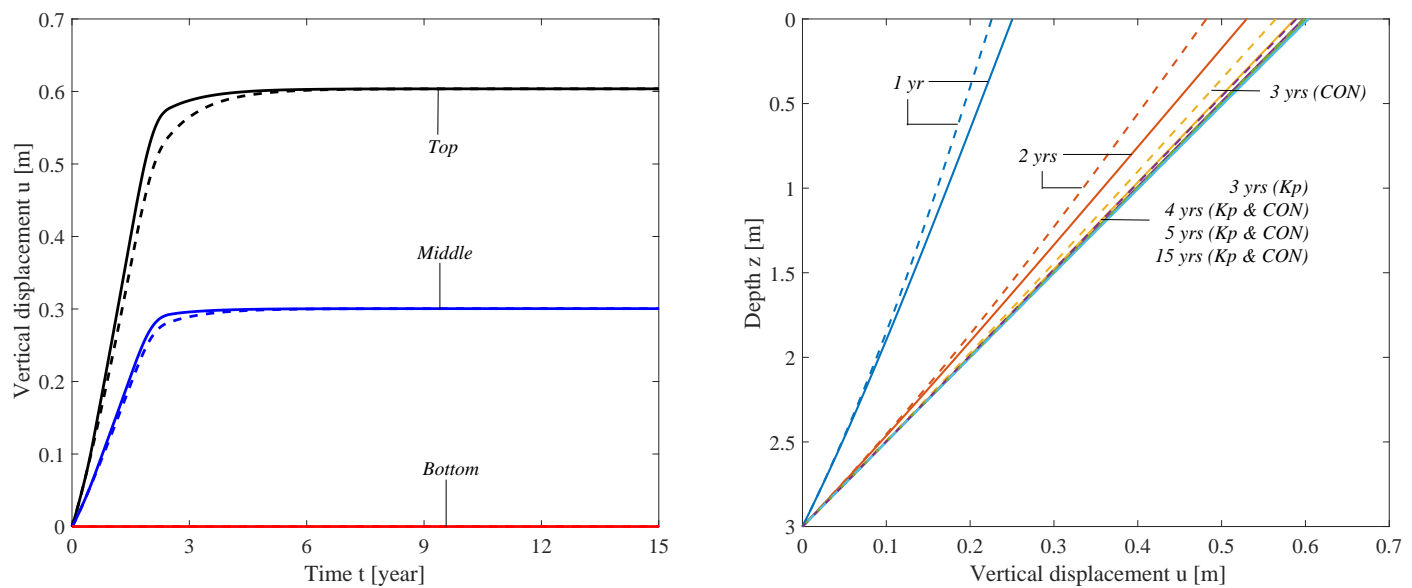

(b) Vertical displacement
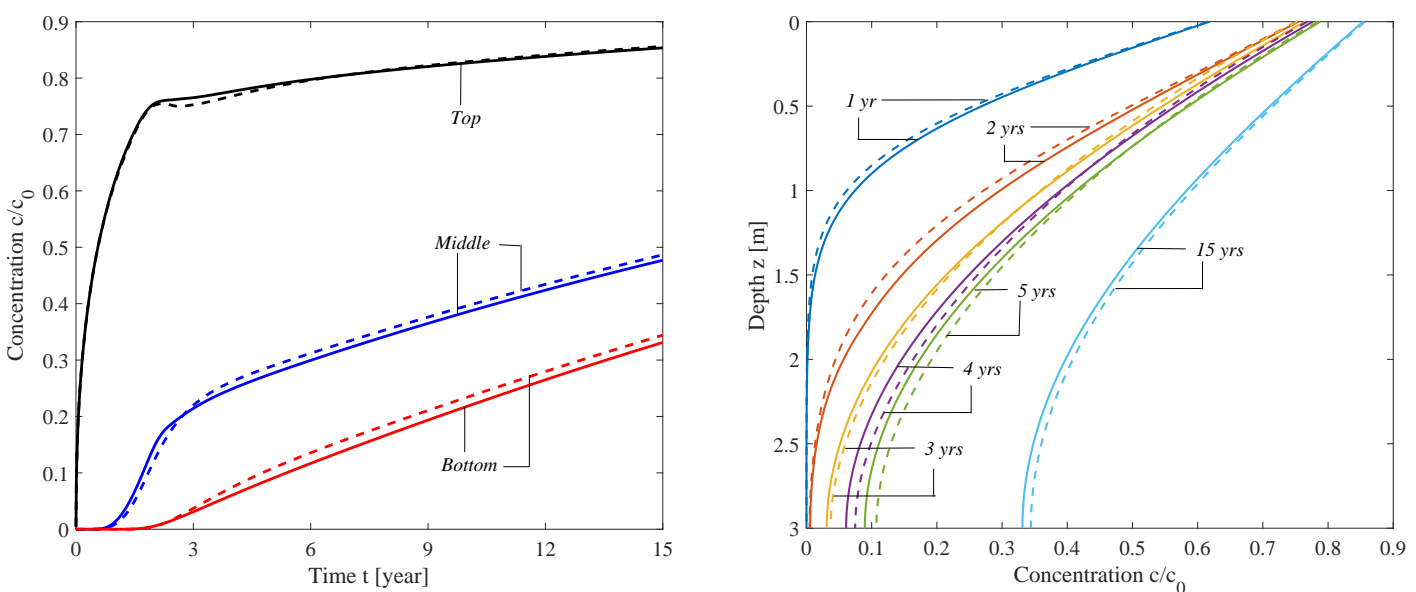

(c) Solute concentration

Figure 7: Distribution of (a) excess pore pressure, (b) vertical displacement and (c) solute transport vs time and depth for Model Kp and Model CON 
year and 2 years confirm the maximum increase at the upper layer, where the pore pressure is harder to dissipate compared to the lower layer. After 3 years, $K$ returns to initial value of $K_{s}$ and remains unchanged through the whole layer to the end of the simulation.

Spatial and temporal changes in $K$ affect the corresponding excess pore pressure $(p)$, as indicated in Fig.7(a). The solid lines describe the results of Model Kp while the dashed lines are the results of Model CON, where $K=K_{s}$. Comparing the two models, some similar trends can be found. For example, $p$ in the upper layer is higher than in the lower layer; $p$ rises rapidly during the loading period then drops gradually after entering the post-loading stage. However, their increasing/dissipation rate and amount are all affected by dynamic $K$. The peak excess pore pressures for Model $\mathrm{Kp}$ at the top point and middle point are $50 \mathrm{kPa} \& 40 \mathrm{kPa}$ respectively, while for Model CON, the highest $p$ is almost double $(100 \mathrm{kPa} \& 75 \mathrm{kPa})$ at the two locations. Moreover, the dynamic conductivity also influences the dissipation rate during the post-loading period. At the top boundary of the clay layer, $p$ has been nearly fully dissipated after 5 years for Model $\mathrm{Kp}$ while a remainder of around $8 \mathrm{kPa}$ exists for the conventional model. These features can be explained by the definition of hydraulic conductivity ; a larger $K$ means the pore fluid is easier to travel within the void, hence accelerates the drainage at the outlet. The dynamic hydraulic conductivity and pore pressure relationship that results in a higher $K$ on the top layer, further leads to a smaller excess pore pressure build up and quicker dissipation.

Compared to the pore pressure, the vertical soil deformation $(u)$ was less influenced by the dynamic conductivity and the final level of soil deformation is not affected at all. The displacement versus time figure (Fig. 7(b)) shows that the largest vertical displacement is $0.6 m$ measured from the top and $0.3 \mathrm{~m}$ at the middle point for both Model Kp and Model CON. The bottom point is assumed to be rigid so no vertical displacement is allowed. However, the rate of soil deformation is affected when $K$ becomes dynamic. The displacement versus depth figure indicates that after 2 years, the top surface has sunk $0.53 \mathrm{~m}$ with dynamic conductivity. However, the conventional model only shows a $0.48 \mathrm{~m}$ downward settlement. Furthermore, the Model Kp predicts that the top surface reaches its final deformation level after 3 years, but Model CON is still $5 \mathrm{~cm}$ short of its final value at that time.

For the volatile pollutant to spread in the soil matrix, part of the solute dissolves in the transient advective flow, which is produced from the soil consolidation process. Meanwhile, 
hydrodynamic dispersion, which is a combined process of molecular diffusion and longitudinal dispersion, takes place. Molecular diffusion is mainly manifested as particles move from an area of high concentration to an area of low concentration and longitudinal diffusion refers to the process whereby solutes are mechanically mixed by velocity variations. Generally speaking, in relatively low speed environments, molecular diffusion dominates the process. For the numerical examples considered here, during the loading period, there is no significant difference at the top point (due to the boundary condition constrain) or the bottom point (since the contaminants have not reached bottom). Nevertheless, a faster contaminant migration can be discovered at the middle point (Fig. 7(c)). Detailed results can be seen from the plot of concentration $\left(c / c_{0}\right)$ versus depth. After 2 years, at a depth of $1.7 \mathrm{~m}$ the contaminant level has reached $10 \%\left(c / c_{0}=0.1\right)$ for Model Kp, while for Model CON, the same contaminant level was reached $10 \mathrm{~cm}$ higher. On the post-loading stage, $K$ recovers to its initial value and the transient excess flow carrying contaminants continues spreading. Due to pore pressure dissipation, the rate of solute transportation reduces in both models. After the pore pressure is fully dissipated, only hydrodynamic dispersion contributes to contaminant transportation. Moreover, according to the outlet boundary condition, the contaminant cannot escape the soil layer, which results in an accumulation at the bottom. Compared to Model CON, Fig. 7(c) reveals that a dynamic $K$ will eventually reduce pollution. For example, the breakthrough time, the time required for the solute concentration to reach $10 \%$ of the referenced concentration at the outlet, takes half a year longer with dynamic conductivity.

\subsection{Dynamic degree of saturation model (Model Srp)}

Here we vary the dynamic degree of saturation (Model Srp), but keep the hydraulic conductivity constant.

Fig. 8 shows that the degree of saturation $\left(S_{r}\right)$ varies between $S_{r}^{0}(0.85)$ and 1 . The dynamic $S_{r}$ happens mainly during the loading period and in the upper layer. As the load acts on the soil layer, the air bubbles are expelled out from the pore fluid and result in a change in the degree of saturation. Fig. 8(a) presents the evolution of $S_{r}$ in the three cut points. Except for the bottom point, degree of saturation at both top point and middle point are greatly changed. Specifically, soil has became fully saturated for nearly 3 years at the top and around 2 years at middle point. 


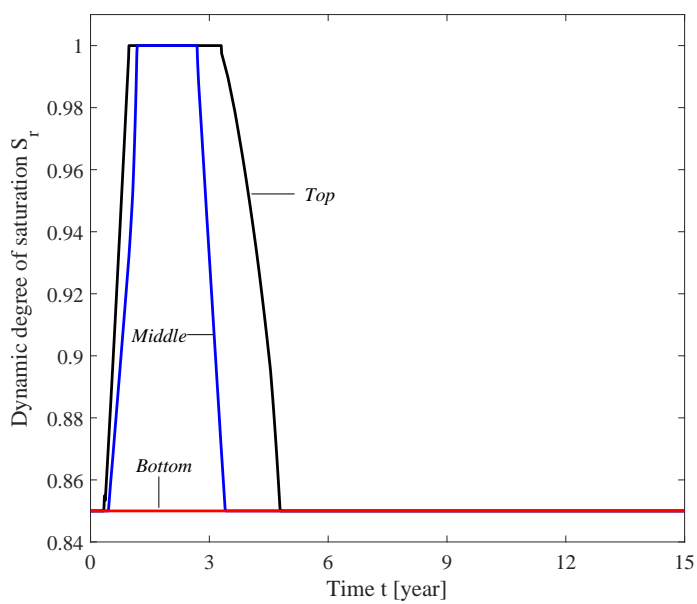

(a) Dynamic degree of saturation vs time

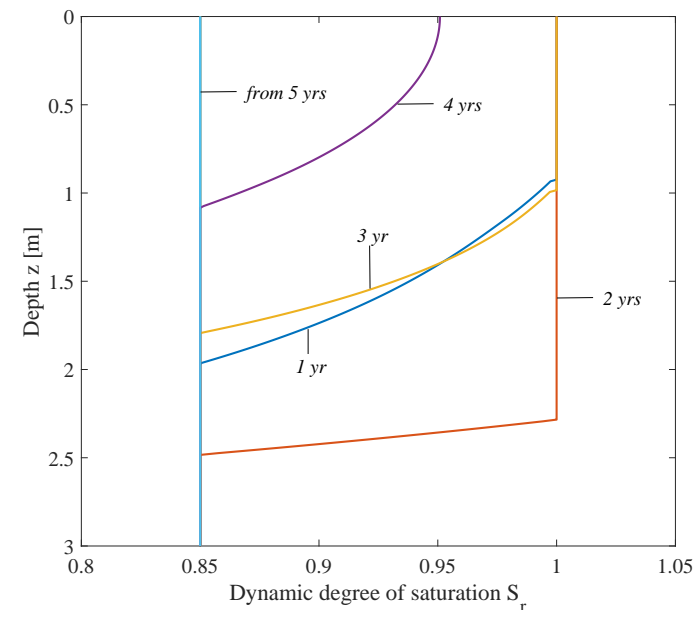

(b) Dynamic degree of saturation distribution

Figure 8: Distribution of dynamic degree of saturation vs time and depth for Model Srp (Type 1)

Due to the constrain of pore pressure at the outlet $S_{r}$ remains unchanged at the level of $S_{r}^{0}$. After 3 years, with the excess pore pressure gradually dissipating, $S_{r}$ reduced. Moreover, Fig. 8(b) shows the vertical distribution of the dynamic $S_{r}$ with different colour lines indicating each time step. After 2 years, the fully saturation zone has reached $2.4 \mathrm{~m}$ down, however after 4 years, the whole clay layer returns to unsaturated status.

The effect of dynamic degree of saturation on the excess pore pressure is plotted in Fig. 9(a), and it is very different from Model Kp. With Model Srp the peak excess pore pressure shows almost no differences compared with Model CON at both top and middle points. However, both excess pore pressure accumulation rate and dissipation rate are affected especially during the period when $S_{r}$ is experiencing either sudden rise ( 0.5 year- 1 year) and drop (3 years - 6 years). This implies that although the change of $S_{r}$ magnitude is small (from 0.85 to 1 ), the rate of change (the additional terms in excess pore pressure governing equations due to $\frac{\partial S_{r}}{\partial t}$ ) contributes to the consolidation process to a certain extent. For Model CON, the excess pore pressure is almost fully dissipated after 6 years, while due to dynamic $S_{r}$, a residue of $10 \mathrm{kPa}$ exits at the middle point. With excess pore pressure expelled out of the soil matrix, the soil deforms. Similar to Model Kp, dynamic $S_{r}$ does not affect the final deformation, but the inlet will eventually drop $0.6 \mathrm{~m}$ (Fig. 9(b)). Due to the reduction of excess pore pressure dissipation rate during Year 3 to Year 6, the soil deforms slightly slower at the this period. For the solute transport, the Model Srp results in a slightly slower solute transport during the loading period 

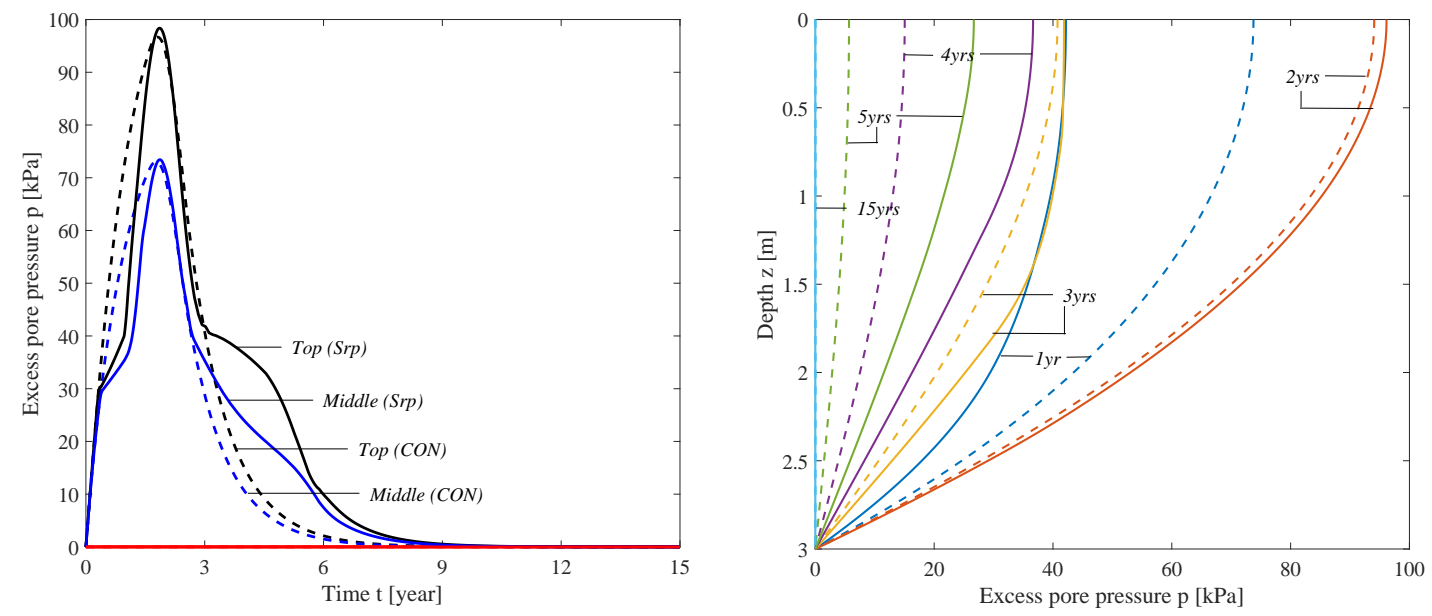

(a) Excess pore pressure
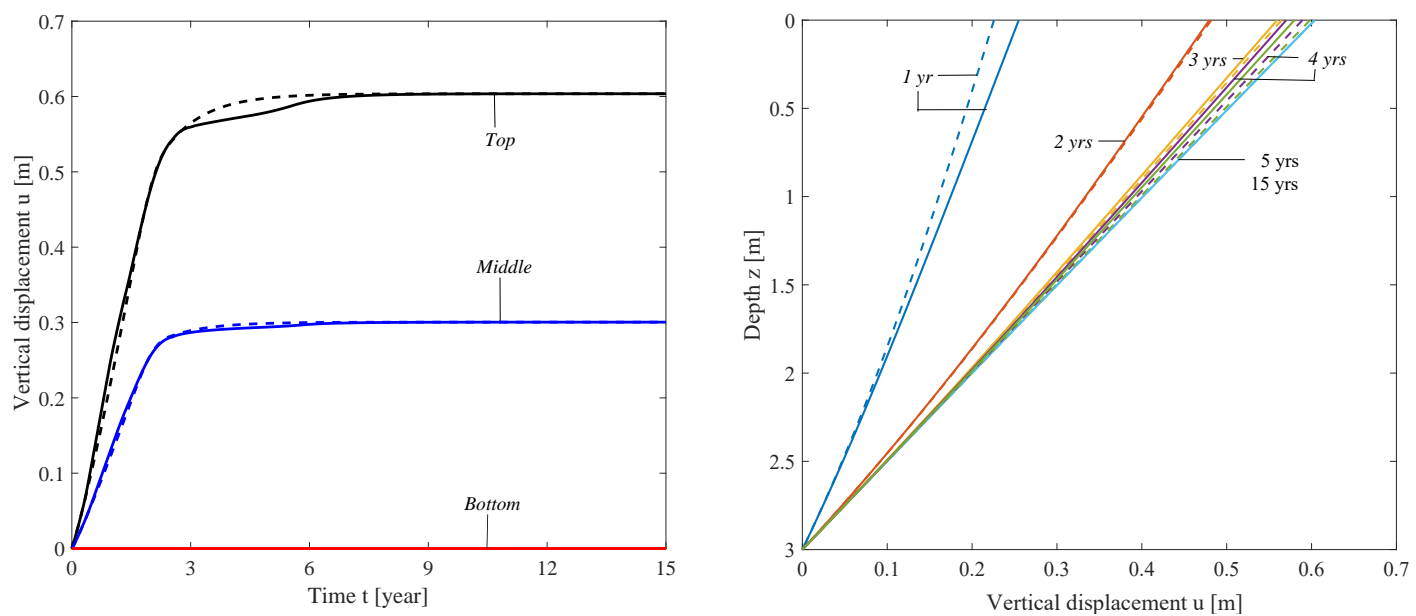

(b) Vertical displacement
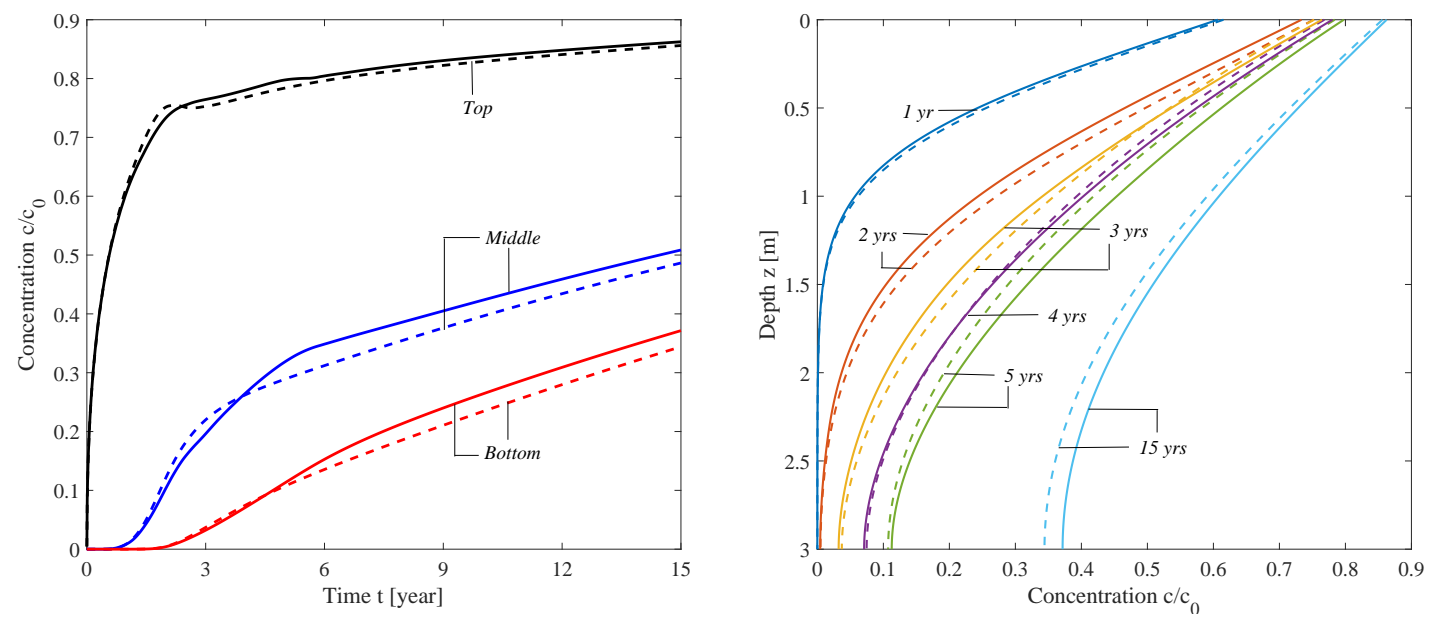

(c) solute concentration

Figure 9: Distribution of (a) excess pore pressure, (b) vertical displacement and (c) solute transport vs time and depth for Model Srp and Model CON 
and a faster spreading on the post-loading stage. Eventually, higher solute concentration can be observed. According to Fig. 9(c), it takes 7.5 years for the outlet to be $20 \%$ contaminated for Model Srp, while with constant $S_{r}$ it takes 1.5 years longer to reach the same contaminated level. At the end of 15 years, the outlet has been polluted to $38 \%$ with dynamic $S_{r}$, while Model CON suggests the final contamination level to be lower than $35 \%$.

\subsection{Dynamic hydraulic conductivity and degree of saturation model (Model Kp+Srp)}

In the dynamic hydraulic conductivity and degree of saturation model (Model Kp+Srp), both hydraulic conductivity and dynamic degree of saturation vary with pore pressure.

Fig. 10 present Model Kp+Srp results for dynamic conductivity and dynamic degree of saturation versus time and depth. According to the dynamic theory, $K, p$ and $S_{r}$ are mutually interacting. As a result, although the same parameters were used in Model Kp+Srp, Model Kp and Model Srp, the evolution of both $K$ and $S_{r}$ are quite different from the previous two dynamic models. Specifically, the peak value of $K$ for the current model is greater than the maximum $K\left(3.5 \times 10^{-9} \mathrm{~m} / \mathrm{s}\right)$ in Model Kp. Moreover, the dynamic $S_{r}$ evolution shows several differences compared with Model Srp, For instance, the fully saturated condition lasts shorter at both top and middle points; after 2 years the fully saturated zone shrinks to $1.5 \mathrm{~m}$ while Model Sp obtained a $2.4 \mathrm{~m}$ saturated zone; when $S_{r}$ recovers back to the level of $S_{r}^{0}$ after 3 years, the top point in Model Srp remains fully saturated.

The dynamic effects of $K$ and $S_{r}$ have been discussed separately in previous sections. Under the dynamic effects of both, compared with Model CON, a reduction of excess pore pressure (Fig. 11(a)) and a slightly faster soil deformation (Fig. 11(b)) can be observed. This is reasonable since the dynamic $S_{r}$ effect is less significant than the dynamic $K$ in term of the soil consolidation process. However, since dynamic $K$ results in a slower solute transport while dynamic $S_{r}$ accelerates the contaminant migration process, for our numerical study case, concentration results for Model $K p+S r p$ eventually shows limited differences with Model CON (Fig. 11(c)). 

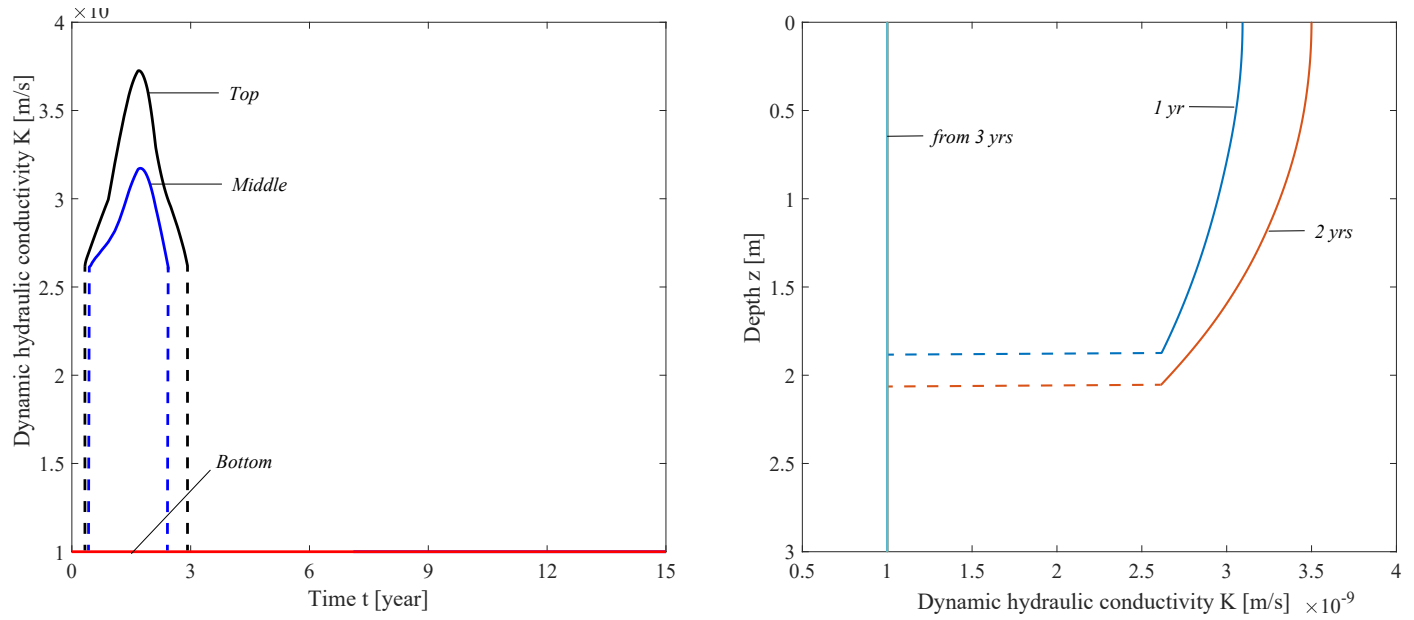

(a) Dynamic hydraulic conductivity
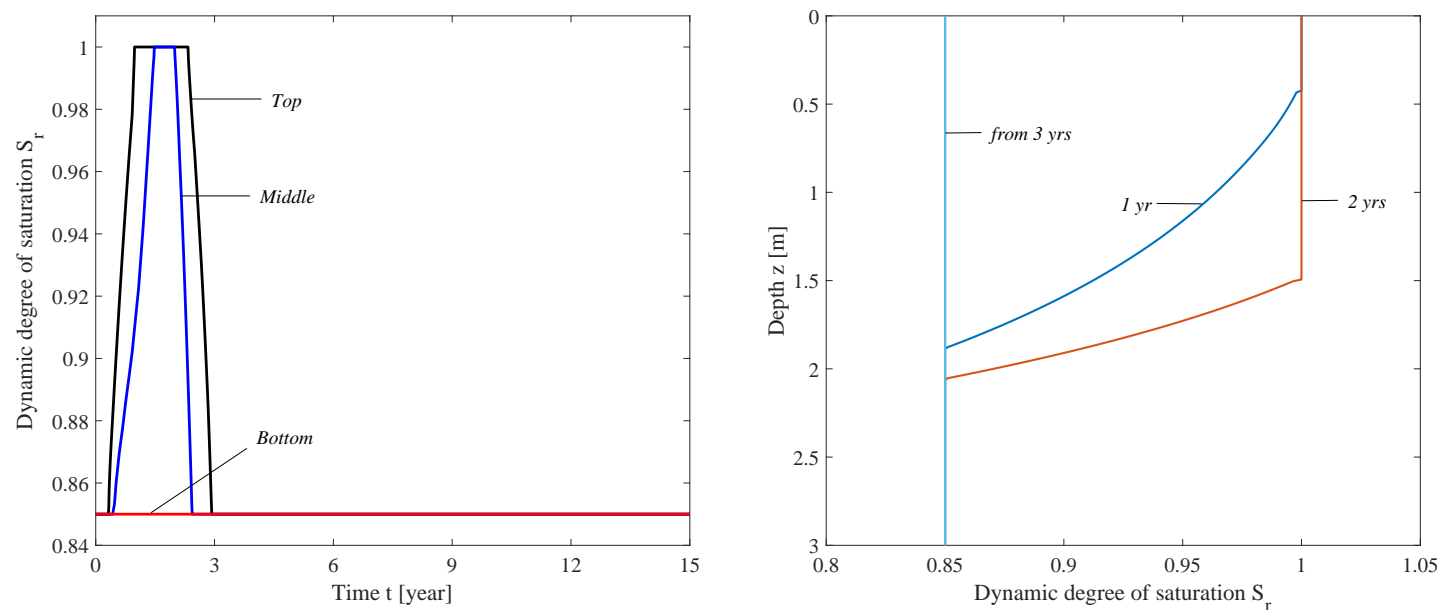

(b) Dynamic degree of saturation

Figure 10: Distribution of (a) dynamic hydraulic conductivity and (b) dynamic degree of saturation vs time and depth for Model Kp+Srp 

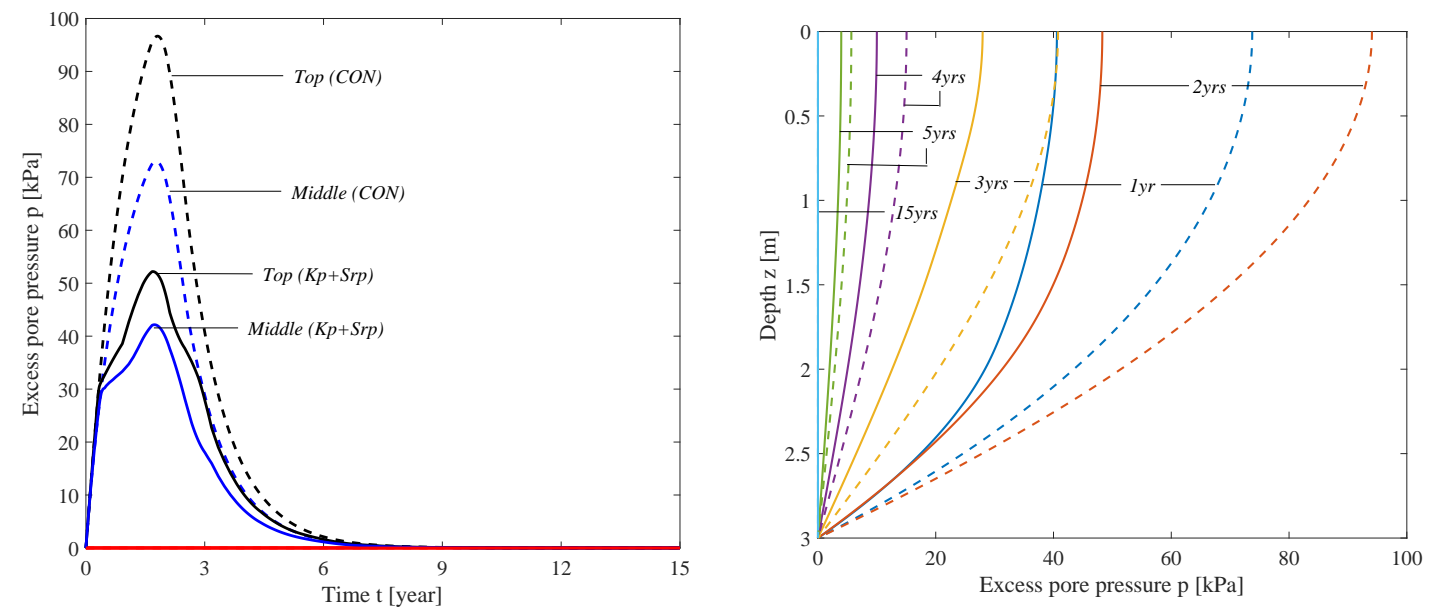

(a) Excess pore pressure
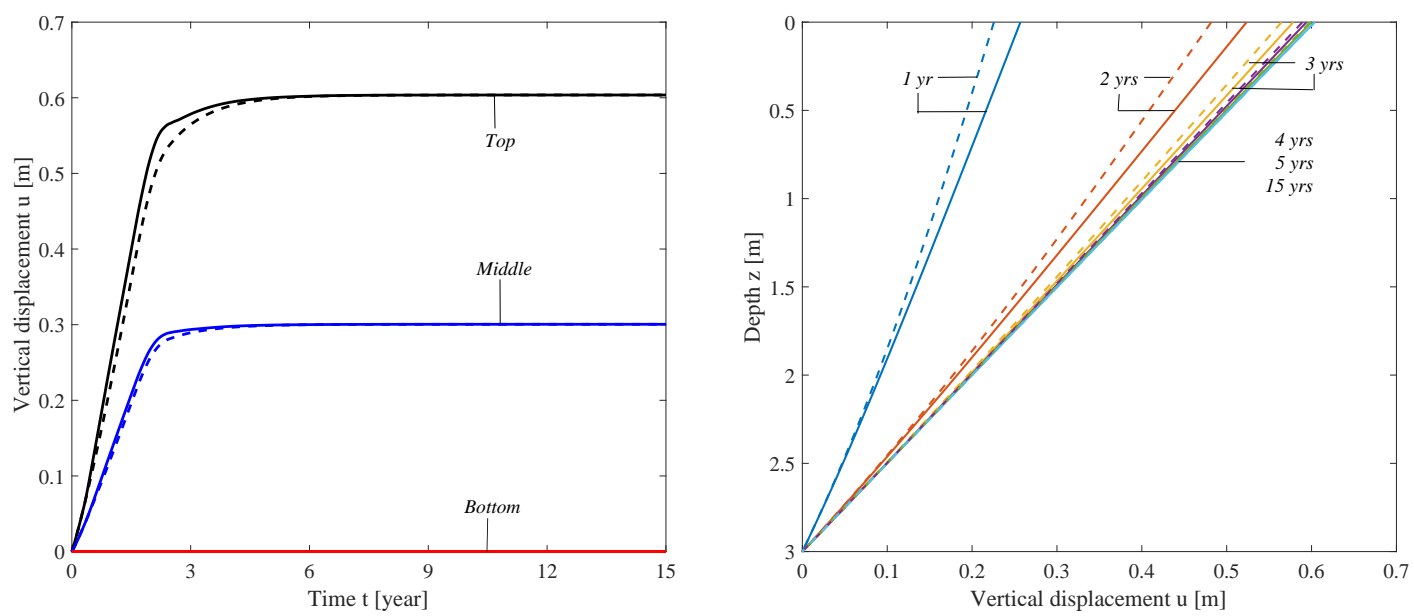

(b) Vertical displacement
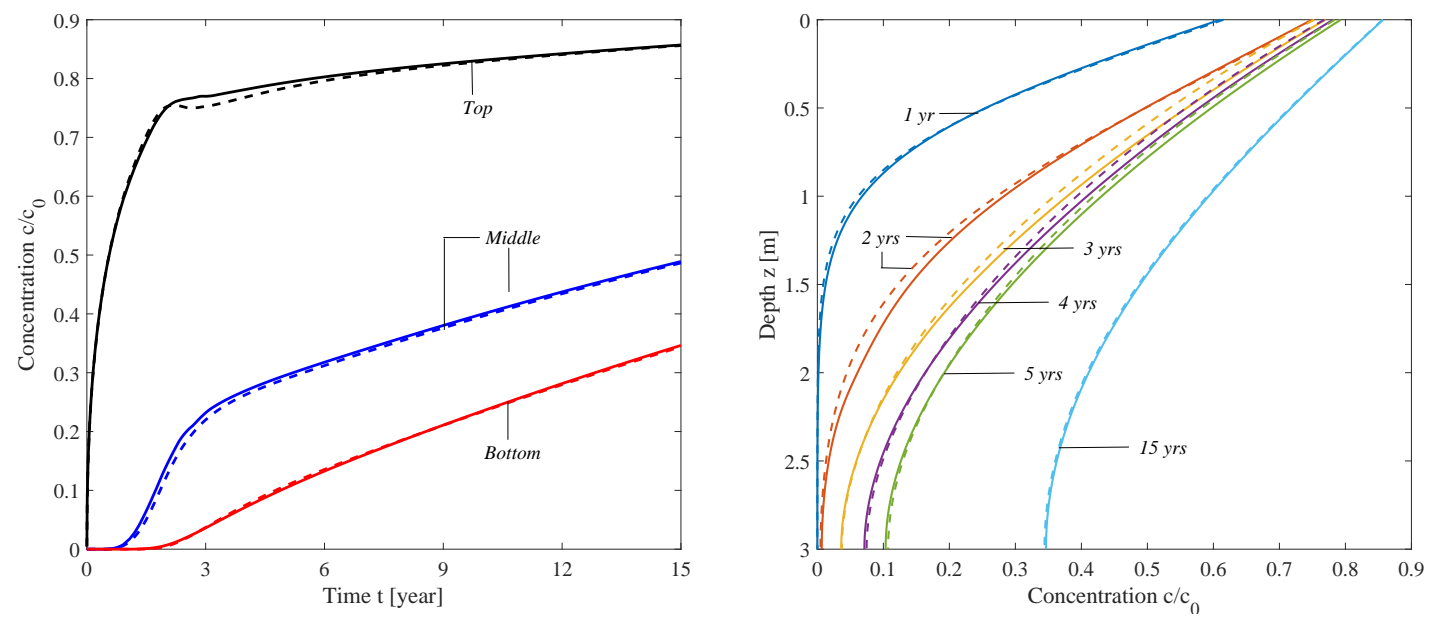

(c) solute concentration

Figure 11: Distribution of (a) excess pore pressure, (b) vertical displacement and (c) solute transport vs time and depth for Model Kp+Srp and Model CON 


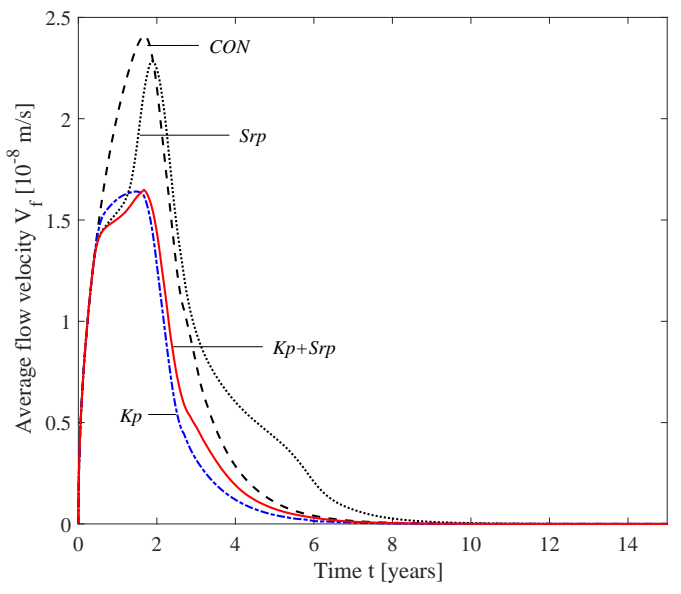

(a) Average flow velocity

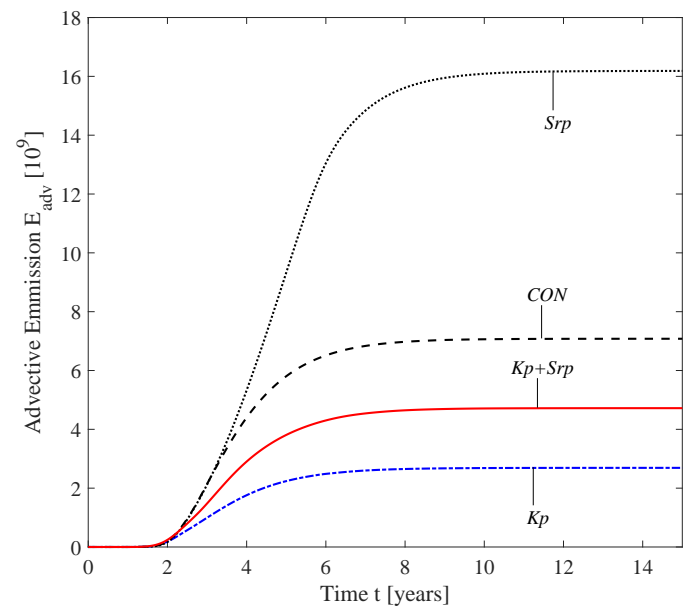

(b) Advective emission

Figure 12: the advective emission and the averaged flow velocity for all models

\subsection{Average flow velocity and advective emission}

Here the average flow velocity $\left(v_{f}\right)$ and the advective emission $\left(E_{a d v}\right)$ for all models are compared to examine the effects of dynamic $K$ and dynamic $S_{r}$ on the consolidation-induced solute transport model.

The average flow velocity at the bottom boundary for each model is plotted in Fig. 12(a). The average flow velocity can be calculated as the sum of Darcy's velocity and the solid phase velocity:

$$
v_{f}=-\frac{K}{S_{r} n^{0} \rho_{w} g} \frac{\partial p}{\partial z}+\frac{\partial u}{\partial t}
$$

The peak $v_{f}$ for all models occurs at around two years, when the post-loading stage begins. The conventional model and dynamic degree of saturation model shows faster transient advective flow, with a peak flow velocity to be around $2.5 \times 10^{-8} \mathrm{~m} / \mathrm{s}$ (Fig. 12(a)). Further, the $v_{f}$ curve for Model $\mathrm{Kp}+\mathrm{Srp}$ does not show much difference to the dynamic $K$ model, but is considerable lower than the other two models. Meanwhile, Model Srp and Model CON generate similar $V_{f}$ trends. Hence, the transient flow reduces speed with the dynamic $K$, however, the varying degree of saturation has less influence on the average advective flow velocity. 
Transient excess flows triggered by soil consolidation shows considerable influence on the solute transport. This effect can be observed from the advective emission $\left(E_{a d v}\right)$ as summarized in Fig. 12(b). $E_{a d v}$ can be calculated as [12]

$$
E_{a d v}=\int_{0}^{t}-\frac{\partial p(\tau)}{\partial z} c(\tau) d \tau
$$

where $\tau$ is the independent variable of integration.

With the assumption of zero concentration gradient at the outlet, no diffusion takes place and only advective flow is allowed. Thus, the advective emission at the bottom boundary refers to the cumulative contaminant mass outflow. Fig. 12(b) presents the advective emission at the bottom boundary for each model. Clearly the advective emissions are significantly different for each dynamic model compared to the conventional model. Specifically, dynamic hydraulic conductivity reduces the advective emission while dynamic degree of saturation tends to increase the emission by a larger amount. However, considering dynamic $K$ and $S_{r}$ simultaneously, Model Kp+Srp model suggest a mitigation of $E_{a d v}$. This feature reveals a coupled effect of dynamic conductivity and degree of saturation so that their effects cannot be simply added. Further, the largest advective emission occurs in Model Srp, which is consistent with the solute concentration result and averaged flow velocity result, that dynamic $S_{r}$ produces a faster solute transport while keeping a relatively fast transient flow rate.

\subsection{Concavity of dynamic degree of saturation function}

In Section 2.2, two expressions were proposed to describe the relationship between dynamic degree of saturation and pore pressure. For both expressions, the degree of saturation increases from $S_{r}^{0}$ to 1 as pore pressure increased. The only difference is that Type 1 function is concave downwards while the Type 2 function is concave up. To study the effect of the concavity to consolidation-induced solute transport, assuming constant hydraulic conductivity, a modified dynamic $S_{r}$ model is used and compared with previous Model Srp.

Using the definition (8), the modified storage equation for dynamic $S_{r}$ Type 2 becomes:

- for $|p| \leq\left|\psi_{a e}\right|$ or $|p|>\left|P_{S r=1}\right|$, same as (14), 
- for $\left|\psi_{a e}\right|<|p| \leq\left|P_{S r=1}\right|$,

$$
\left(S_{r} n^{0} \beta+n^{0} \alpha e^{\alpha \psi_{a e}+\alpha p}\right) \frac{\partial p}{\partial t}+S_{r} \frac{\partial^{2} u}{\partial z \partial t}=\frac{K}{\rho_{w} g}\left(\frac{\partial^{2} p}{\partial z^{2}}\right)-n^{0} \alpha e^{\alpha \psi_{a e}+\alpha p} \frac{\partial u}{\partial t} \frac{\partial p}{\partial z} .
$$

The modified solute transport equation are:

- for $|p| \leq\left|\psi_{a e}\right|$ or $|p|>\left|P_{S r=1}\right|$, same as (17)

- for $\left|\psi_{a e}\right|<|p| \leq\left|P_{S r=1}\right|$,

$$
\begin{aligned}
{\left[S_{r} n^{0}+\left(1-n^{0}\right) \rho_{s} K_{d}\right] \frac{\partial c}{\partial t} } & =\frac{\partial^{2} c}{\partial z^{2}}\left(S_{r} n^{0} D_{m}-\alpha_{L} \frac{K}{\rho_{w} g} \frac{\partial p}{\partial z}\right) \\
& +\frac{\partial c}{\partial z}\left\{n^{0} \alpha e^{\alpha \psi_{a e}+\alpha p}\left(D_{m} \frac{\partial p}{\partial z}-\alpha_{L} \frac{\partial p}{\partial t}-\alpha_{L} \frac{\partial u}{\partial t} \frac{\partial p}{\partial z}\right)\right. \\
& -\alpha_{L} S_{r} n^{0} \beta \frac{\partial p}{\partial t}-\alpha_{L} S_{r} \frac{\partial^{2} u}{\partial z \partial t} \\
& +\frac{\alpha_{L} \beta K}{\rho_{w} g}\left(\frac{\partial p}{\partial z}\right)^{2}+S_{r} D_{m}\left(1-n^{0}\right) \frac{\partial^{2} u}{\partial z^{2}} \\
& \left.+\frac{K}{\rho_{w} g} \frac{\partial p}{\partial z}-\left[S_{r} n^{0}+\left(1-n^{0}\right) \rho_{s} K_{d}\right] \frac{\partial u}{\partial t}\right\} \\
& +c\left[S_{r} n^{0} \beta \frac{\partial p}{\partial t}-\beta \frac{K}{\rho_{w} g}\left(\frac{\partial p}{\partial z}\right)^{2}+S_{r} n^{0} \beta \frac{\partial u}{\partial t} \frac{\partial p}{\partial z}\right]
\end{aligned}
$$

Note that the force balance equation remains the same as previous models.

The distribution of dynamic degree of saturation versus time and depth are shown in Fig. 13. Solid lines indicate dynamic $S_{r}$ Type 2 results while dashed lines are from the previous Model Srp (Type 1 dynamic $S_{r}$ theory). In generally, the Type 2 shows a more pronounced change in degree of saturation dynamics. Specifically, as shown in Fig. 13(b), at year 5, the whole soil layer returns the original $S_{r}$ level of 0.85 for Type 1, while Type 2 shows a higher $S_{r}$ in the upper layer (around $z=0.3 \mathrm{~m}$ ). The fully saturated zone extents deeper with dynamic $S_{r}$ Type 2, so that, for example, the fully saturated zone is nearly half meter thicker in year 2 and 1 meter thicker in year 3 . Moreover, Type 2 dynamic $S_{r}$ results in a slightly longer fully-saturated period at both top and middle points.

Fig. 14 (a) indicates that, while the Type 2 model has an impact on pore pressure distribution for a short period (during year 5 to year 9), there are no significant differences on the peak pore pressure values. Regardless of the path how $S_{r}$ reaches unity, it takes 9 years for pore pressure to 


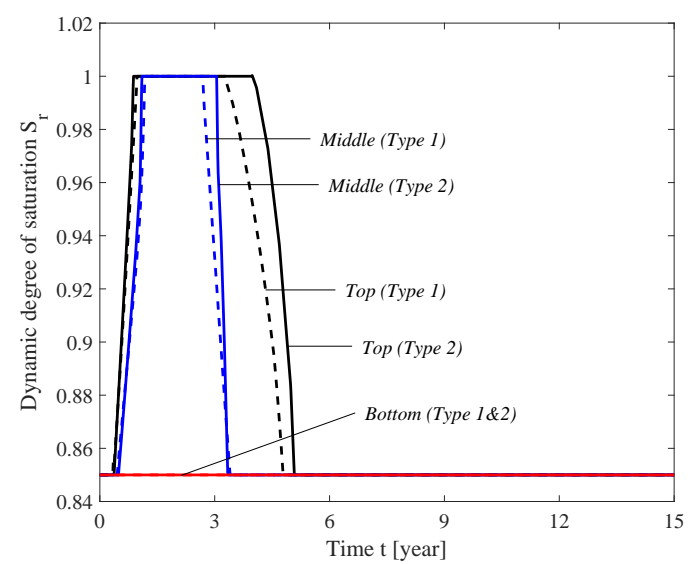

(a) Dynamic degree of saturation vs time

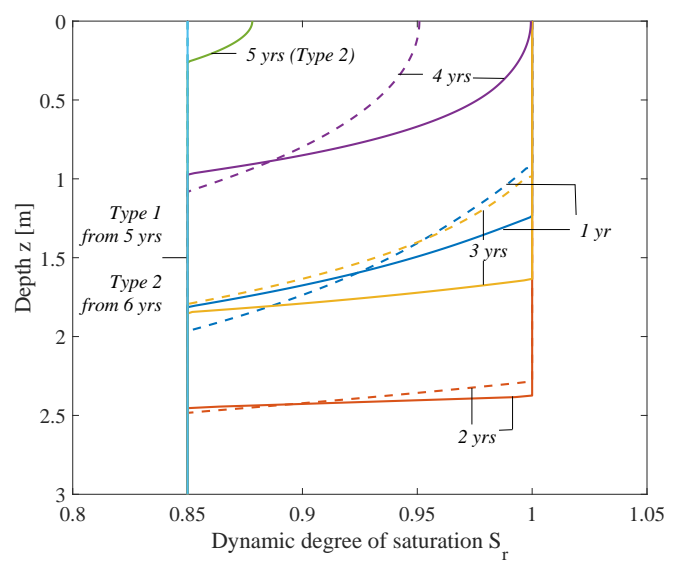

(b) Dynamic degree of saturation distribution

Figure 13: Distribution of dynamic degree of saturation vs time and depth for Model Srp, comparison between Type 1(dashed lines) and Type 2(solid line)

be fully dissipated for both models. Further, Fig. 14(b) indicates similar vertical displacement distribution for the two models showing that the soil deformation is hardly influenced by the concavity of dynamic $S_{r}$ formulation. Consequently, the effect of concavity of dynamic $S_{r}$ on solute concentration is limited (Fig. 14(c)).

\subsection{Parametric study for varied air-entry value}

As discussed in Section 3, the air-entry value $\left(\psi_{a e}\right)$ is an important parameter of the criterion for judging whether hydraulic conductivity and degree of saturation are dynamic. Only in the situation where excess pore pressure is greater than the air-entry value, $K$ and $S_{r}$ are considered to be varying with respect to the pore pressure. Consequently, the effects of varied $\psi_{a e}$ to the consolidation-induced solute transport is particularly of interest and presented in this section. Fig. 15-17 show the parametric study results for Model Kp, Model Srp and Model Kp+Srp respectively. In each figure, solid lines, dashed lines and dotted lines denote the simulation results when $\psi_{a e}$ equals $20 \mathrm{kPa}, 30 \mathrm{kPa}$ and $39 \mathrm{kPa}$ assuming a clayey type of soil.

In general, $\psi_{a e}$ alters the time when $K$ and $S_{r}$ become dynamic and affects the magnitudes of these parameters. Smaller $\psi_{a e}$ implies that pore pressure is more easier to excess the air-entry value, so that the longer dynamic period can be observed. For Model Kp, with smaller $\psi_{a e}$ (20 $\mathrm{kPa}$ ), Fig. 15(a) indicates the hydraulic conductivity starts to increasing from 0.25 year, and 

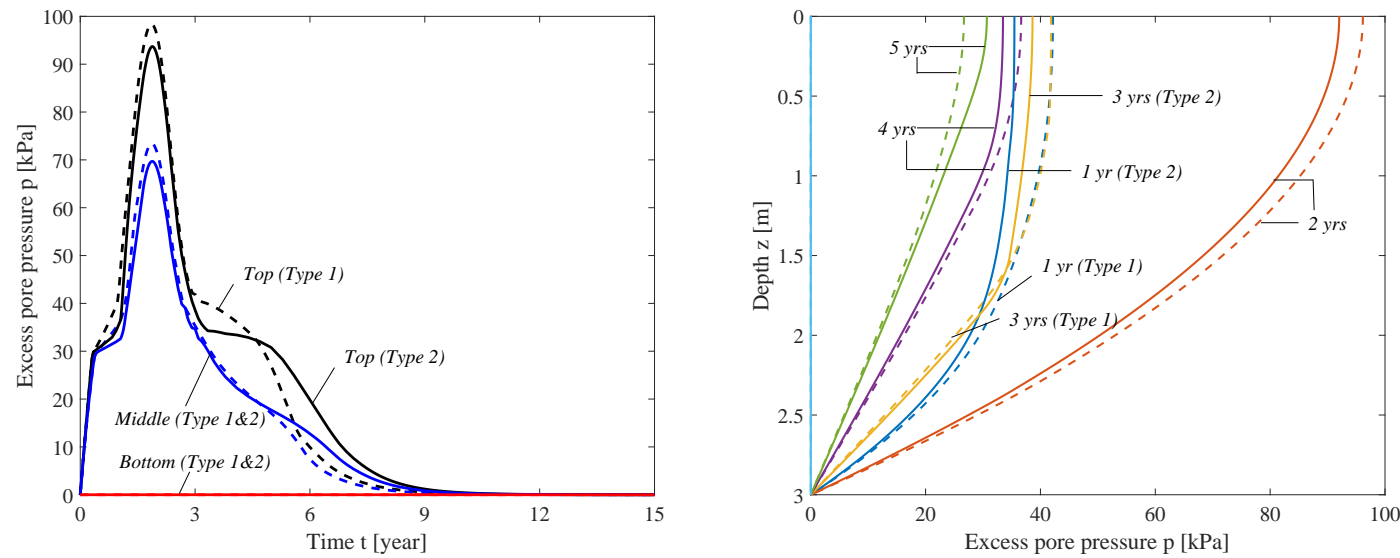

(a) Excess pore pressure
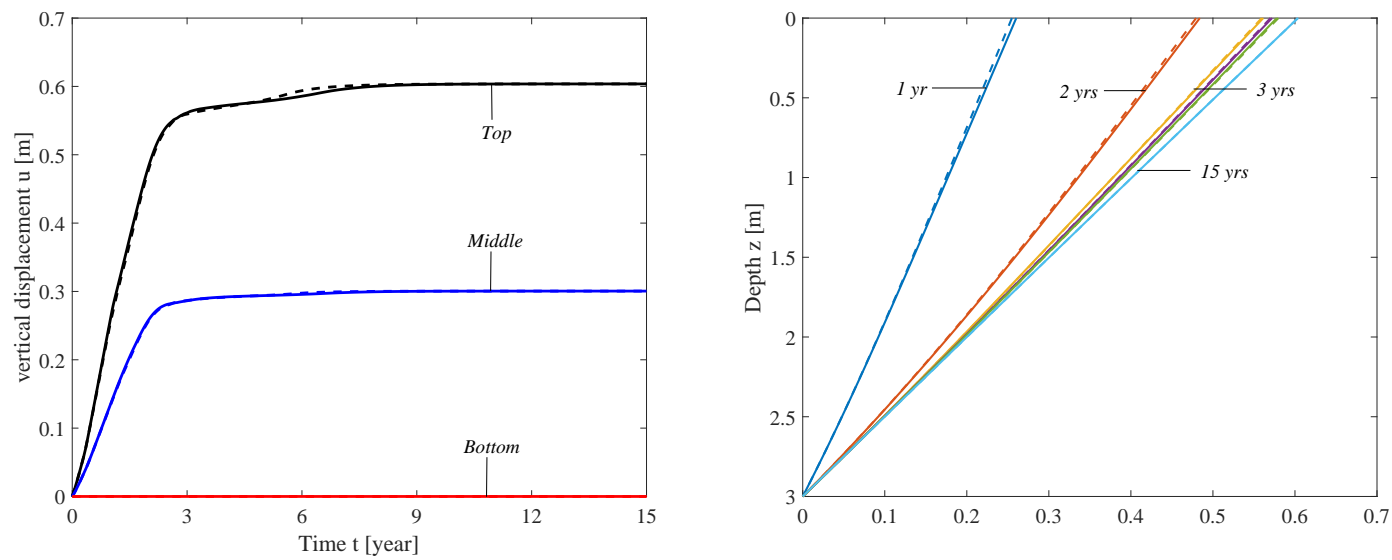

(b) Vertical displacement
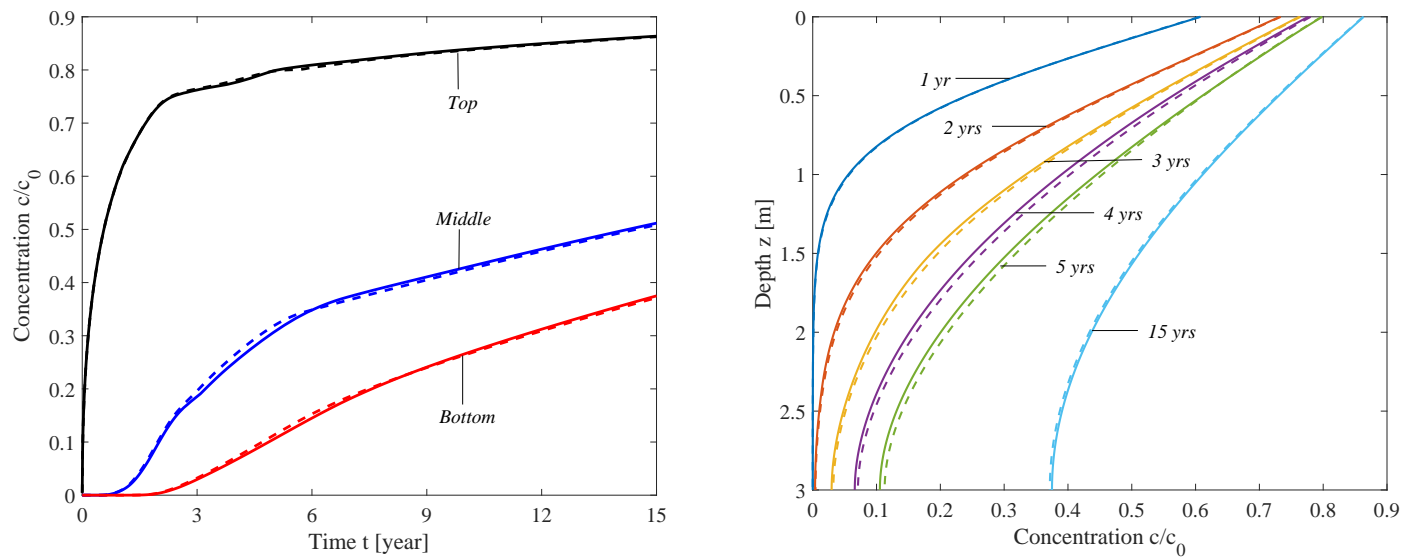

(c) Solute concentration

Figure 14: Distribution of (a) excess pore pressure, (b) vertical displacement and (c) solute transport vs time and depth for Model Srp, comparison between Type 1(dashed lines) and Type 2(solid line) 


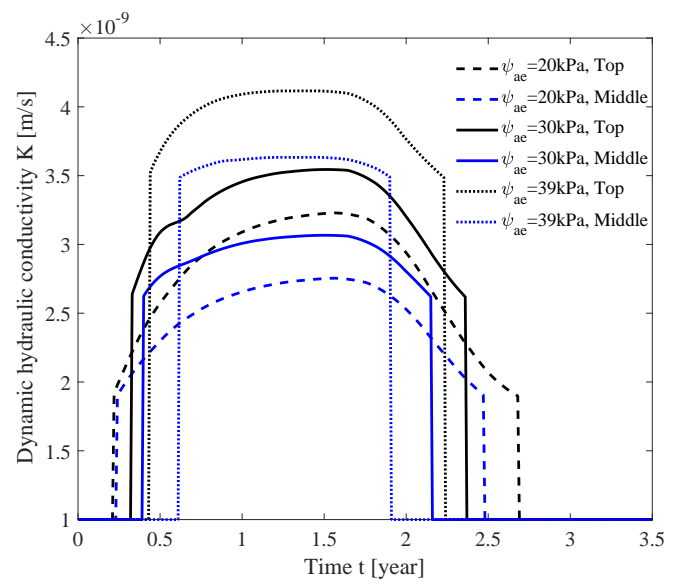

(a) Dynamic hydraulic conductivity vs time

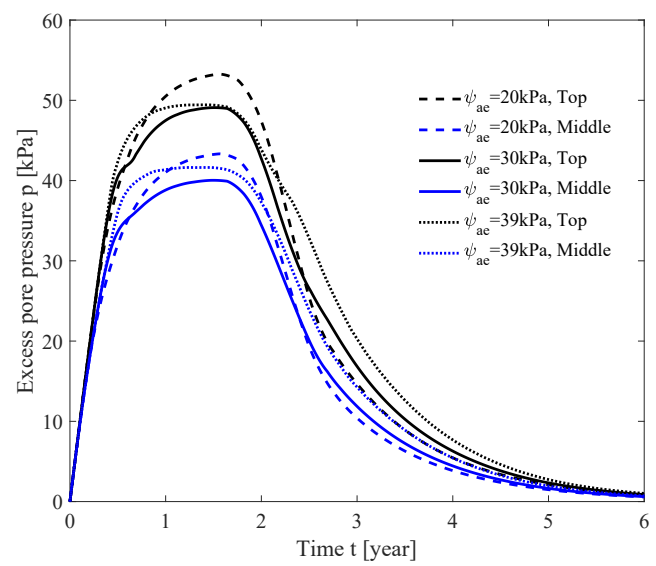

(b) Excess pore pressure vs time

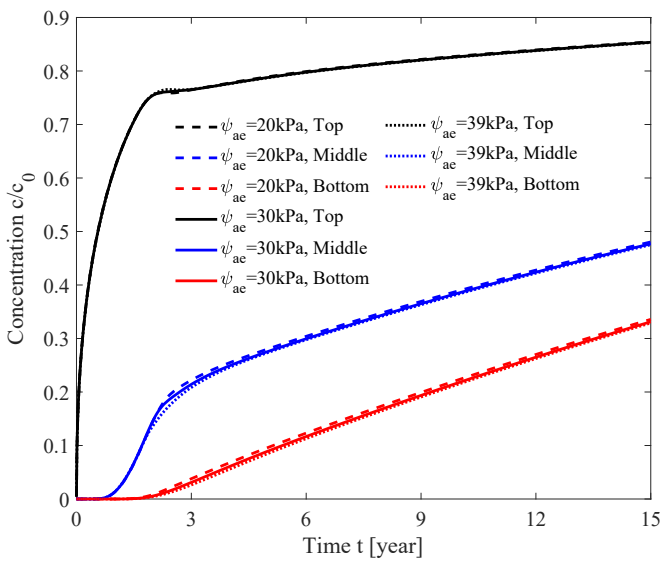

(c) Solute concentration vs time

Figure 15: Distribution of (a) dynamic hydraulic conductivity (b) excess pore pressure and (c) solute concentration vs time for Model Kp with varied $\psi_{a e}$

the dynamic period ends at 3.5 year at the top point and 2.5 year at the middle point. With the largest air entry value of $39 \mathrm{kPa}$, the hydraulic conductivity dynamic period is shorten to around 1.8 years for middle point and around 2 years at top. It is important to point out that, the outlet boundary (bottom) is assumed to allow free drainage of the excess pore fluid $\left(p^{e}=0\right)$ so that pore pressure will never exceed the air entry value, and this feature further leads to constant $K$ and $S_{r}$ at bottom through out the whole simulation time. In addition to the influence of dynamic period, another impact on the magnitude of the dynamic $K$ can be conclude that larger $\psi_{a e}$ results in lower $K$ at both top and middle points. With the combined effects of both, the excess pore pressure distribution (Fig. 15(b)) shows the lowest peak value occurs when $\psi_{a e}=30 \mathrm{kPa}$, 


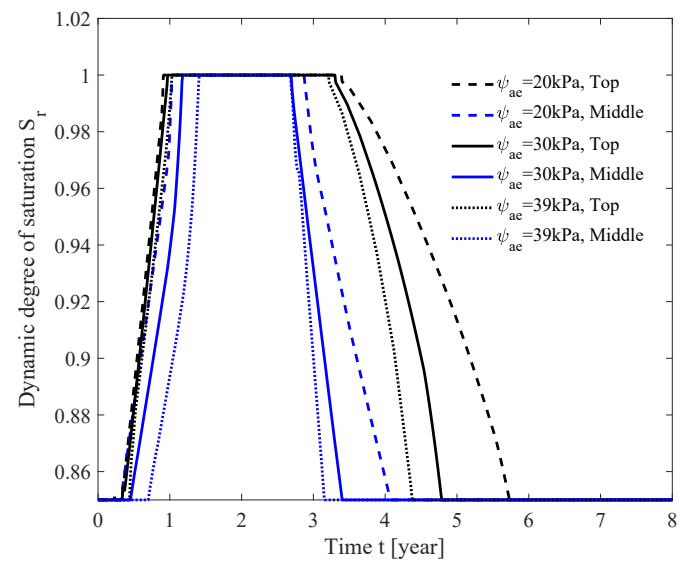

(a) Dynamic degree of saturation vs time

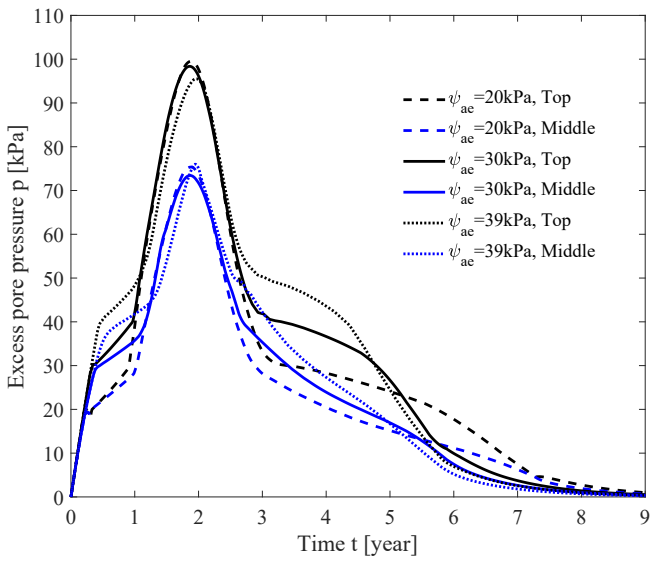

(b) Excess pore pressure vs time

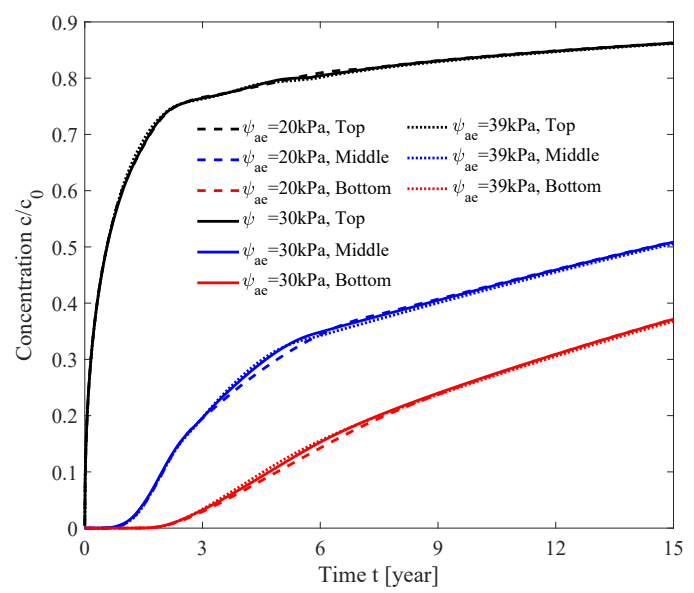

(c) Solute concentration vs time

Figure 16: Distribution of (a) dynamic degree of saturation (b) excess pore pressure and (c) solute concentration vs time for Model Srp with varied $\psi_{a e}$

Fig. 16 presents the dynamic degree of saturation, excess pore pressure and solute concentration distribution for Model Srp with varied $\psi_{a e}$. When conducting the parametric study, the value $P_{S r=1}$ needs to be adjusted for each air-entry value $\left(P_{S r=1}=30 \mathrm{kPa} \& 49 \mathrm{kPa}\right.$ for $\psi_{a e}=20 \mathrm{kPa}$ $\& 39 \mathrm{kPa})$. Although the starting point of dynamic degree of saturation is less affected, longer 


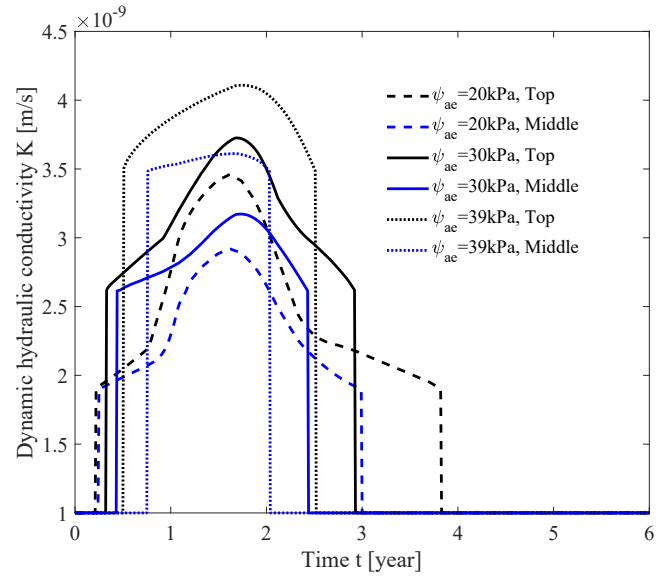

(a) Dynamic hydraulic conductivity vs time

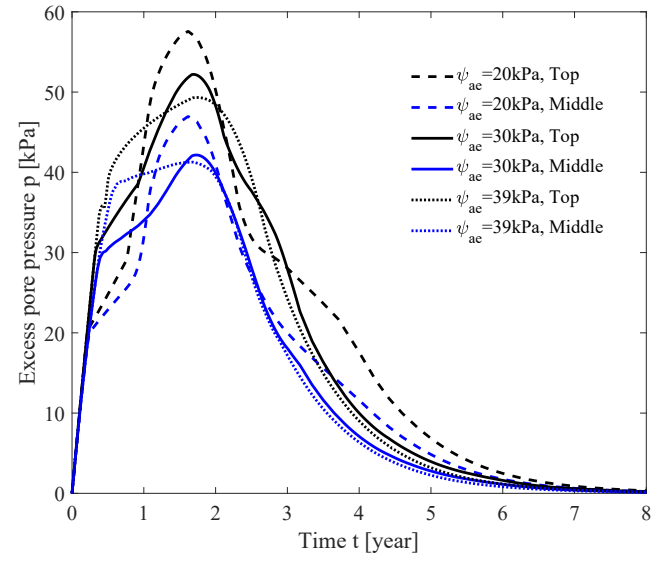

(c) Excess pore pressure vs time

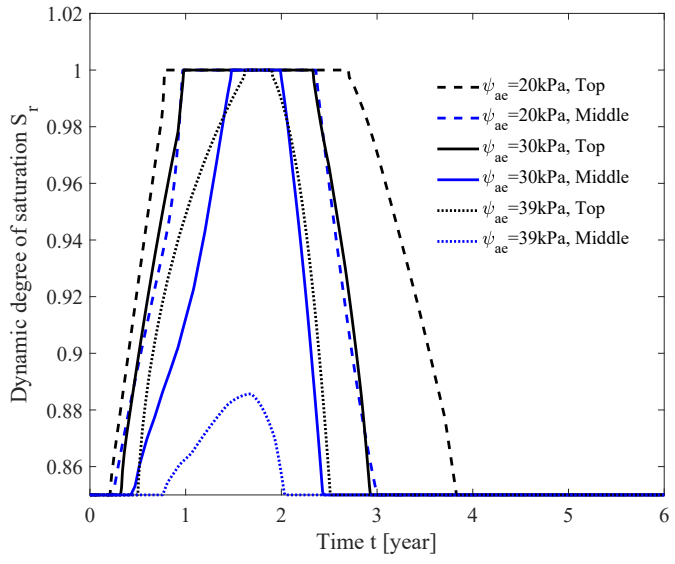

(b) Dynamic degree of saturation vs time

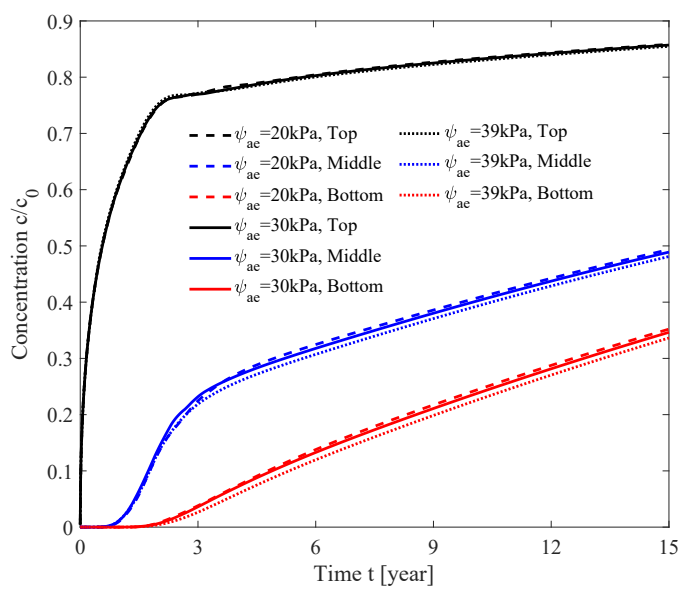

(d) Solute concentration vs time

Figure 17: Distribution of (a) dynamic hydraulic conductivity (b) dynamic degree of saturation (c) excess pore pressure and (d) solute concentration vs time for Model Kp+Srp with varied $\psi_{a e}$

In addition, the parametric study is also conducted for Model $\mathrm{Kp}+\mathrm{Srp}$, parameter $\mathbb{I}_{S r=1}$ is set to be as same as listed in parametric study of Model Srp. As shown in Fig. 17(a)\&(b), dynamic period for both hydraulic conductivity and degree of saturation is reduced compared to Model Kp and Model Srp. The peak values of $K$ are similar as in Model Kp, while compared to Model 
Srp, differences can be observed. For example, at the middle point when $\psi_{a e}=39 \mathrm{kPa}$, the peak degree of saturation only reaches 0.88 , while in previous dynamic degree of saturation model, a 1.5 year fully saturation period exists at the same location. The lowest excess pore pressure occurs when $\psi_{a e}=39 \mathrm{kPa}$, and the peak value exists when $\psi_{a e}=39 \mathrm{kPa}$ at around $60 \mathrm{kPa}$ (Fig. 17(c)). This peak excess pore pressure is slightly higher than Model Kp and much lower than peak $p^{e}$ in Model Srp, which implies that the hydraulic conductivity plays a more significant role in governing the excess pore pressure dissipation compared with degree of saturation. Lastly, according to Fig. 17(d), larger air-entry value generally contributes to a slower contaminant migration and leads to a later break through time. Recall that both Model Kp and Model Srp show limited impact on solute concentration, the fully dynamic model finally demonstrates the effects of varied air-entry value to the consolidation-induced solute migration.

\section{Conclusions}

In this study, three dynamic models were established, one for dynamic hydraulic conductivity (Model Kp), one for dynamic degree of saturation (Model Srp) and the third dynamic model for both (Model Kp+Srp). Due to the lack of field measurements and experimental data, model validation was conducted between previous numerical studies $[10,12]$ and present dynamic model while control the excess pore pressure to be always less than air entry value. The dynamic model results were compared with the conventional model to see the dynamic effects on the consolidation-induced solute transport. Following that, an alternative dynamic $S_{r}$ model is proposed and compared with Model Srp to study the effects of the concavity of pore pressure related dynamic degree of saturation function on consolidation-induced solute transport. Additionally, parametric study was performed to investigate the model sensitivity to the air-entry value.

In general, the consideration of dynamic hydraulic conductivity greatly affects the consolidation process and slightly reduces the consolidation-induced solute transport speed. the dynamic degree of saturation, on the other hand, results in an acceleration of contaminant migration. It is important to include the dynamic soil parameters especially when pore pressure is large and the air-entry value is low. Furthermore, based on the simulation results, more conclusions can be drawn: 
(1) Considering $K$ and $S_{r}$ to be dynamic and varying with respect to pore pressure, both $K$ and $S_{r}$ increased, but only during the period when pore pressure exceeding the air-entry value. Dynamic $K$ and $S_{r}$ may only happen for certain depth within the clay layer.

(2) The Dynamic hydraulic conductivity model (Model Kp) results in less excess pore pressure build up, faster soil deformation and slightly slower solute transport.

(3) The Dynamic degree of saturation model (Model Srp) does not affect to peak excess pore pressure but would accelerate contaminant migration.

(4) The dynamic hydraulic conductivity and degree of saturation model (Model Kp+Srp) combines Model Kp and Model Srp. The numerical simulation is compared with the conventional model and the dynamic model suggests less excess pore pressure build up, but shows no significant differences on solute transport.

(5) Dynamic $K$ and dynamic $S_{r}$ are coupled effects and can not be simply added up. To correctly consider dynamic effects for both simultaneously, Model Kp+Srp is applicable.

(6) Although the dynamic degree of saturation has certain effects on consolidation-induced solute transport, the dynamic path of $S_{r}$ has limited influence on contaminant migration.

(7) Higher air-entry value results in shorter dynamic periods for both $K$ and $S_{r}$. Although varied $\psi_{a e}$ shows less influence on the dynamic degree of saturation model, higher $\psi_{a e}$ leads to slower solute transport when considering dynamic constitutive relationship of hydraulic conductivity.

This study introduces novel ideas by considering dynamic changes of soil parameters. By extending the conventional solute transport in deformable unsaturated porous media, the fully dynamic model now is able to incorporate the fluid compressibility, dynamic fluid density, dynamic soil porosity, dynamic hydraulic conductivity as well as the dynamic degree of saturation. The application of incorporating dynamic soil parameters in soil response is not limited to the landfill case as presented in this study, more examples could be made such as seabed response or marine structure stability [32]. 


\section{Appendix A. Derivation of fluid storage equation with dynamic hydraulic conductivity and degree of saturation}

Mass conservation for pore fluid is presented as:

$$
\frac{\partial}{\partial t}\left(S_{r} n \rho_{w}\right)=-\frac{\partial}{\partial z}\left(S_{r} n \rho_{w} v_{f}\right)
$$

in which $S_{r}$ refers degree of saturation, $n$ is the porosity, $v_{f}$ denotes the flow velocity in the voids and $\rho_{w}$ is the density of pore fluid.

The well known Darcy's Law can be written as:

$$
S_{r} n\left(v_{f}-v_{s}\right)=-\frac{K}{\rho_{w} g} \frac{\partial p}{\partial z}
$$

where $p$ represents the excess pore pressure $K$ indicates hydraulic conductivity and $v_{s}$ refers to the velocity of the solid.

Keeping in mind that $K, S_{r}$ and $\rho_{w}$ are all dynamic and substituting (A.2) into (A.1) gives the relationship shown as:

$$
\rho_{w} \frac{\partial S_{r} n}{\partial t}+S_{r} n \frac{\partial \rho_{w}}{\partial t}=-\frac{\partial}{\partial z}\left[\rho_{w}\left(-\frac{K}{\rho_{w} g} \frac{\partial p}{\partial z}+S_{r} n v_{s}\right)\right]
$$

Expending (A.3) leads to:

$$
\rho_{w} \frac{\partial S_{r} n}{\partial t}+S_{r} n \frac{\partial \rho_{w}}{\partial t}=\frac{1}{g} \frac{\partial}{\partial z}\left(K \frac{\partial p}{\partial z}\right)-S_{r} n v_{s} \frac{\partial \rho_{w}}{\partial z}-\rho_{w} \frac{\partial}{\partial z}\left(S_{r} n v_{s}\right)
$$

Although the soil is partially saturated, the degree of saturation is assumed to be relatively high, so that the air is embedded in pore water in the form of bubbles. The fluid compressibility $(\beta)$ can then be expressed interns of degree of saturation as [27]:

$$
\beta=\frac{S_{r}}{K_{w 0}}+\frac{1-S_{r}+r_{h} S_{r}}{P_{a}+P_{0}}
$$


in which $K_{w 0}$ is the pore water bulk modulus $(1000 \mathrm{MPa}), r_{h}$ is the volumetric fraction of dissolved air within pore water (0.02), $P_{a}$ and $P_{0}$ are the gauge air pressure and the atmosphere pressure $\left(P_{a}+P_{0}=100 \mathrm{kPa}\right)$.

The fluid density $\left(\rho_{w}\right)$ is considered to be changing with pore pressure, which implies the spatial and time variation of fluid density as:

$$
\begin{aligned}
& \frac{\partial \rho_{w}}{\partial t}=\beta \rho_{w} \frac{\partial p}{\partial t}, \\
& \frac{\partial \rho_{w}}{\partial z}=\beta \rho_{w} \frac{\partial p}{\partial z} .
\end{aligned}
$$

Hence, (A.4) becomes to:

$$
S_{r} \frac{\partial n}{\partial t}+n \frac{\partial S_{r}}{\partial t}+S_{r} n \beta\left(\frac{\partial p}{\partial t}+v_{s} \frac{\partial p}{\partial z}\right)=\frac{1}{\rho_{w} g} \frac{\partial}{\partial z}\left(K \frac{\partial p}{\partial z}\right)-S_{r} \frac{\partial n v_{s}}{\partial z}-n v_{s} \frac{\partial S_{r}}{\partial z}
$$

Considering the mass conservation of solid phase, the temporal derivative of porosity can be expressed as [12]:

$$
\frac{\partial n}{\partial t}=\frac{\partial v_{s}}{\partial z}-\frac{\partial n v_{s}}{\partial z}
$$

Further, the assumption of relatively small deformation implies the relationship that:

$$
\left|\frac{\partial p}{\partial t}\right| \gg\left|v_{s} \frac{\partial p}{\partial z}\right|
$$

where the velocity of solid phase can be calculated as $\frac{\partial u}{\partial t}$.

Combining (A.8), (A.9) and (A.10), the governing equation for excess pore pressure field that is applicable to the condition of dynamic hydraulic conductivity and dynamic degree of saturation is proposed as:

$$
n \frac{\partial S_{r}}{\partial t}+S_{r} n \beta \frac{\partial p}{\partial t}+S_{r} \frac{\partial^{2} u}{\partial z \partial t}=\frac{K}{\rho_{w} g}\left(\frac{\partial^{2} p}{\partial z^{2}}\right)+\frac{1}{\rho_{w} g} \frac{\partial K}{\partial z} \frac{\partial p}{\partial z}-n \frac{\partial u}{\partial t} \frac{\partial S_{r}}{\partial z} .
$$




\section{Appendix B. Derivation of solute transport equation with dynamic hydraulic conductiv- ity and degree of saturation}

For the governing equation of solute concentration field, conservation of solute mass in fluid and solid phase were considered, so that the solute transport equation can be written as:

$$
\frac{\partial}{\partial t}\left(S_{r} n c\right)+\frac{\partial}{\partial t}\left[(1-n) c_{s}\right]=-\frac{\partial}{\partial z}\left[S_{r} n\left(-D \frac{\partial c}{\partial z}+v_{f} c\right)+(1-n) v_{s} c_{s}\right]
$$

where $c$ and $c_{s}$ refer to the solute concentration in fluid and solid phase, respectively, and $D$ denotes the hydrodynamic dispersion coefficient.

Under the assumption of compressible fluid and incompressible soil particle and combining with mass conservation of pore fluid and soil particles, Zhang et al.[12] suggested the transport equation to be written as:

$$
S_{r} n \frac{\partial c}{\partial t}+(1-n) \frac{\partial c_{s}}{\partial t}=\frac{\partial}{\partial z}\left(S_{r} n D \frac{\partial c}{\partial z}\right)-S_{r} n v_{f} \frac{\partial c}{\partial z}-(1-n) v_{s} \frac{\partial c_{s}}{\partial z}+S_{r} n \beta\left(\frac{\partial p}{\partial t}+v_{f} \frac{\partial p}{\partial z}\right) c
$$

By considering linear sorption:

$$
c_{s}=\rho_{s} K_{d} c
$$

in which $K_{d}$ describes the partitioning of the contaminant, and substitute $v_{f}$ and $v_{s}$ as introduced in Appendix A into (B.2), the governing equation can be expressed as:

$$
\begin{aligned}
{\left[S_{r} n+(1-n) \rho_{s} K_{d}\right] \frac{\partial c}{\partial t} } & =S_{r} n D \frac{\partial^{2} c}{\partial z^{2}} \\
& +\frac{\partial c}{\partial z}\left[\frac{\partial}{\partial z}\left(S_{r} n D\right)+\frac{K}{\rho_{w} g} \frac{\partial p}{\partial z}-S_{r} n \frac{\partial u}{\partial t}-(1-n) \rho_{s} K_{d} \frac{\partial u}{\partial t}\right] \\
& +c\left[S_{r} n \beta \frac{\partial p}{\partial t}-\beta \frac{K}{\rho_{w} g}\left(\frac{\partial p}{\partial z}\right)^{2}+S_{r} n \beta \frac{\partial u}{\partial t} \frac{\partial p}{\partial z}\right]
\end{aligned}
$$

To incorporate the dynamic degree of saturation in the derivation process, chain rule is applied so that: 


$$
\frac{\partial}{\partial z}\left(S_{r} n D\right)=S_{r} n \frac{\partial D}{\partial z}+S_{r} D \frac{\partial n}{\partial z}+D n \frac{\partial S_{r}}{\partial z}
$$

$$
D=D_{m}+\alpha_{L}\left(v_{f}-v_{s}\right)
$$

Furthermore, the spatial variation can be calculated as [12] :

$$
\frac{\partial n}{\partial z}=\frac{(1-n)^{2}}{1-n^{0}} \frac{\partial^{2} u}{\partial z^{2}}
$$

537 where $n^{0}$ refers to the initial soil porosity.

Substituting (B.5), (B.6) and (B.7) into (B.4), the governing equation becomes to:

$$
\begin{aligned}
{\left[S_{r} n+(1-n) \rho_{s} K_{d}\right] \frac{\partial c}{\partial t} } & =S_{r} n D_{m} \frac{\partial^{2} c}{\partial z^{2}}+S_{r} n \alpha_{L}\left(v_{f}-v_{s}\right) \frac{\partial^{2} c}{\partial z^{2}} \\
& +\frac{\partial c}{\partial z}\left\{S_{r} n \alpha_{L} \frac{\partial\left(v_{f}-v_{s}\right)}{\partial z}+S_{r} D_{m} \frac{(1-n)^{2}}{1-n^{0}} \frac{\partial^{2} u}{\partial z^{2}}\right. \\
& +S_{r} \alpha_{L}\left(v_{f}-v_{s}\right) \frac{\partial n}{\partial z}+D_{m} n \frac{\partial S_{r}}{\partial z}+n \alpha_{L}\left(v_{f}-v_{s}\right) \frac{\partial S_{r}}{\partial z} \\
& \left.+\frac{K}{\rho_{w} g} \frac{\partial p}{\partial z}-\left[S_{r} n+(1-n) \rho_{s} K_{d}\right] \frac{\partial u}{\partial t}\right\} \\
& +c\left[S_{r} n \beta \frac{\partial p}{\partial t}-\beta \frac{K}{\rho_{w} g}\left(\frac{\partial p}{\partial z}\right)^{2}+S_{r} n \beta \frac{\partial u}{\partial t} \frac{\partial p}{\partial z}\right]
\end{aligned}
$$

Utilising the produce rule, the following relationship can be obtained, i.e.,

$$
S_{r} n \frac{\partial\left(v_{f}-v_{s}\right)}{\partial z}+S_{r}\left(v_{f}-v_{s}\right) \frac{\partial n}{\partial z}=\frac{\partial}{\partial z}\left[S_{r} n\left(v_{f}-v_{s}\right)\right]-n\left(v_{f}-v_{s}\right) \frac{\partial S_{r}}{\partial z}
$$

Combining with Darcy's law (A.2), (B.8) now becomes: 


$$
\begin{aligned}
{\left[S_{r} n+(1-n) \rho_{s} K_{d}\right] \frac{\partial c}{\partial t} } & =\frac{\partial^{2} c}{\partial z^{2}}\left(S_{r} n D_{m}-\alpha_{L} \frac{K}{\rho_{w} g} \frac{\partial p}{\partial z}\right) \\
& +\frac{\partial c}{\partial z}\left\{\alpha_{L} \frac{\partial}{\partial z}\left(-\frac{K}{\rho_{w} g} \frac{\partial p}{\partial z}\right)+S_{r} D_{m} \frac{(1-n)^{2}}{1-n^{0}} \frac{\partial^{2} u}{\partial z^{2}}\right. \\
& \left.+D_{m} n \frac{\partial S_{r}}{\partial z}+\frac{K}{\rho_{w} g} \frac{\partial p}{\partial z}-\left[S_{r} n+(1-n) \rho_{s} K_{d}\right] \frac{\partial u}{\partial t}\right\} \\
& +c\left[S_{r} n \beta \frac{\partial p}{\partial t}-\beta \frac{K}{\rho_{w} g}\left(\frac{\partial p}{\partial z}\right)^{2}+S_{r} n \beta \frac{\partial u}{\partial t} \frac{\partial p}{\partial z}\right]
\end{aligned}
$$

Now, recall the updated storage equation (A.11) and apply the product rule, the only term which might be affected by the dynamic hydraulic conductivity can be replaced as:

$$
\begin{aligned}
\frac{\partial}{\partial z}\left(-\frac{K}{\rho_{w} g} \frac{\partial p}{\partial z}\right) & =-\frac{1}{\rho_{w} g} \frac{\partial}{\partial z}\left(K \frac{\partial p}{\partial z}\right)+K \frac{\partial p}{\partial z}\left[\frac{\partial}{\partial z}\left(-\frac{1}{\rho_{w} g}\right)\right] \\
& =-S_{r} \frac{\partial^{2} u}{\partial z \partial t}-n \frac{\partial S_{r}}{\partial t}-S_{r} n \beta \frac{\partial p}{\partial t}-n \frac{\partial u}{\partial t} \frac{\partial S_{r}}{\partial z}+\beta \frac{K}{\rho_{w} g}\left(\frac{\partial p}{\partial z}\right)^{2} .
\end{aligned}
$$

Moreover, based on the discussion in [12], it is reasonable to approximate $n$ to a constant initial soil porosity $\left(n^{0}\right)$ at this stage. Hence, the final governing equation for solute concentration field is presented as:

$$
\begin{aligned}
{\left[S_{r} n^{0}+\left(1-n^{0}\right) \rho_{s} K_{d}\right] \frac{\partial c}{\partial t} } & =\frac{\partial^{2} c}{\partial z^{2}}\left(S_{r} n^{0} D_{m}-\alpha_{L} \frac{K}{\rho_{w} g} \frac{\partial p}{\partial z}\right) \\
& +\frac{\partial c}{\partial z}\left\{-n^{0} \alpha_{L} \frac{\partial S_{r}}{\partial t}-n^{0} \alpha_{L} \frac{\partial u}{\partial t} \frac{\partial S_{r}}{\partial z}+D_{m} n^{0} \frac{\partial S_{r}}{\partial z}\right. \\
& -\alpha_{L} S_{r} n^{0} \beta \frac{\partial p}{\partial t}-S_{r} \alpha_{L} \frac{\partial^{2} u}{\partial z \partial t}+S_{r} D_{m}\left(1-n^{0}\right) \frac{\partial^{2} u}{\partial z^{2}} \\
& \left.+\frac{\alpha_{L} \beta K}{\rho_{w} g}\left(\frac{\partial p}{\partial z}\right)^{2}+\frac{K}{\rho_{w} g} \frac{\partial p}{\partial z}-\left[S_{r} n^{0}+\left(1-n^{0}\right) \rho_{s} K_{d}\right] \frac{\partial u}{\partial t}\right\} \\
& +c\left[S_{r} n^{0} \beta \frac{\partial p}{\partial t}-\beta \frac{K}{\rho_{w} g}\left(\frac{\partial p}{\partial z}\right)^{2}+S_{r} n^{0} \beta \frac{\partial u}{\partial t} \frac{\partial p}{\partial z}\right] .
\end{aligned}
$$

\section{References}

[1] D. A. Barry, Modelling contaminant transport in the subsurface: Theory and computer programs, In: Modelling Chemical Transport in Soil: Natural and Applied Contaminants, edited by Ghadiri, H. and Rose, C. W., Chapter 3, Lewis Publishers, Boca Raton, Florida, USA, 1992, 105-144. 
[2] J. Bear, Dynamics of Fluids in Porous Media, Elsevier Scientific Publishing Company, New York, (1972).

[3] Q. Wang, H. Zhan, On different numerical inverse Laplace methods for solute transport problems, Advances in Water Resources 75 (2015) 80-92.

[4] F. Boso, A. Bellin, M. Dumbser, Numerical simulations of solute transport in highly heterogeneous formations: A comparison of alternative numerical schemes, Advances in Water Resources 52 (2013) 178-189.

[5] J. R. Craig, A. J. Rabideau, Finite difference modeling of contaminant transport using analytic element flow solutions, Advances in Water Resources 29 (7) (2006) 1075-1087.

[6] M. Rolle, D. Hochstetler, G. Chiogna, P. K. Kitanidis, P. Grathwohl, Experimental investigation and pore-scale modeling interpretation of compound-specific transverse dispersion in porous media, Transport in Porous Media 93 (3) (2012) 347-362.

[7] A. Alshawabkeh, N. Rahbar, T. C. Sheahan, G. Tang, Volume change effects in solute transport in clay under consolidation, Geo Jordan 2004 : Advances in Geotechnical Engineering with Emphasis on Dams, Highway Materials, and Soil Improvement, 1 (2004) $105-115$.

[8] L. J. Potter, C. Savvidou, R. E. Gibson, Consolidation and pollutant transport associated with slurried mineral waste disposal, The 1st International Conference on Environmental Geotechnics, Edmonton, Canada (1994) 525-530.

[9] D. W. Smith, One-dimensional contaminant transport through a deforming porous medium : theory and a solution for a quasi-steady-state problem, International Journal for Numerical and Analytical Methods in Geomechanics 24 (8) (2000) 693-722.

[10] G. P. Peters, D. W. Smith, Solute transport through a deforming porous medium, International Journal for Numerical and Analytical Methods in Geomechanics 26 (7) (2002) $683-717$. 
[11] M. A. Biot, General theory of three-dimensional consolidation, Journal of Applied Physics 26 (2) (1941) 155-164.

[12] H. Zhang, D.-S. Jeng, B. R. Seymour, D. A. Barry. L. Li, Solute transport in partiallysaturated deformable porous media: Application to a landfill clay liner, Advances in Water Resources 40 (2012) 1-10.

[13] H. Pu, P. J. Fox, Model for coupled large strain consolidation and solute transport in layered soils, International Journal of Geomechanics,ASCE 16 (2) (2016) 04015064.

[14] S. Wu, D.-S. Jeng, Numerical modeling of solute transport in deformable unsaturated layered soil, Water Science and Engineering 10 (3) (2017) 184-196.

[15] F. Tavenas, P. Jean, P. Leblond, S. Leroueil, The permeability of natural soft clays. Part II: Permeability characteristics, Canadian Geotechnical Journal 20 (4) (1983) 645-660.

[16] A. Al-Tabbaa, D. M. Wood, Some measurements of the permeability of kaolin, Géotechnique 37 (4) (1987) 499-514

[17] R. P. Chapuis, Predicting the saturated hydraulic conductivity of sand and gravel using effective diameter and void ratio, Canadian Geotechnical Journal 41 (5) (2004) 787-795.

[18] G. Bird, Molecular gas dynamics and the direct simulation monte carlo of gas flows, Clarendon, Oxford 508 (1994) 128.

[19] F. Civan, C. S. Rai, C. H. Sondergeld, Shale-gas conductivity and diffusivity inferred by improved formulation of relevant retention and transport mechanisms, Transport in Porous Media 86 (3) (2011) 925-944.

[20] W. R. Gardner, Some steady-state solutions of the unsaturated moisture flow equation with application to evaporation from a water table, Soil Science 85 (4) (1958) 228-232.

[21] L. Wu, L. M. Zhang, Analytical solution to 1d coupled water infiltration and deformation in unsaturated soils, International Journal for Numerical and Analytical Methods in Geomechanics 33 (6) (2009) 773-790. 
[22] C. McKee, A. Bumb, The importance of unsaturated flow parameters in designing a hazardous waste site, in: Hazardous Waste and Environmental Emergencies, Hazardous Materials Control Research Institute National Conference, Houston, Tex, (1984) 12-14.

[23] D. Gallipoli, S. Wheeler, M. Karstunen, Modelling the variation of degree of saturation in a deformable unsaturated soil., Géotechnique 53 (1) (2003) 105-112.

[24] M. T. V. Genuchten, A closed form equation for predicting the hydraulic conductivity of unsaturated soils, Soil Science Society of America Journal 44 (1980) 892-898.

[25] D. Hillel, Environmental Soil Physics: Fundamentals, Applications, and Environmental Considerations, Elsevier, 1998.

[26] R. Hills, I. P. Hudson, P. J. Wierenga, Modeling one-dimensional infiltration into very dry soils, Water Resources Research 25 (1989) 1271-1282.

[27] D. G. Fredlund, H. Rahardjo, Soil Mechanics for Unsaturated Soils, Wiley, 1993.

[28] N. Sivakugan, Inadequacy in the Classification of Coarse-Grained Soils, ASTM International, 1990.

[29] G. B. Wallace, W. C. Otto, Differential settlement at selfridge air force base, Journal of the Soil Mechanics and Foundations Division, ASCE 90 (5) (1964) 197-220.

[30] J. R. Philip, Theory of infiltration, Advances in Hydroscience, (5) (1969) 215-296.

[31] T. L. Zhan, C. W. Ng, Analytical analysis of rainfall infiltration mechanism in unsaturated soils, International Journal of Geomechanics, ASCE 4 (4) (2004) 273-284.

[32] S. Wu, D. -S. Jeng, Effects of dynamic soil permeability on the wave-induced seabed response around a buried pipeline, Ocean Engineering, 186 (2019) 106132. 